\title{
Tensor Functional Topology on Woronowicz Categories
}

\author{
Wendy Lowen ${ }^{1}$ (D) $\cdot$ Joris Mestdagh ${ }^{1}$
}

Received: 19 December 2015 / Accepted: 28 December 2015 / Published online: 29 January 2016

(C) The Author(s) 2016. This article is published with open access at Springerlink.com

\begin{abstract}
Functional topology is concerned with developing topological concepts in a category endowed with certain axiomatically defined classes of morphisms (Clementino et al. 2004). In this paper, we extend functional topology to a monoidal framework, replacing categorical pullbacks by pullbacks relative to the monoidal structure (which itself replaces the product) or more generally relative to a relation on the category (Janelidze, Appl. Categ. Structures, 17(4),351-371, 2009). Our main application is to the opposite Woronowicz category of $C^{*}$-algebras. In this category a natural class of proper morphisms yields the unital algebras as compact objects. When restricted to the commutative $C^{*}$-algebras, we recover exactly the morphisms induced by proper continuous maps of locally compact Hausdorff spaces. We further endow this category with a factorization system and investigate the precise relation with the proper maps, building on an approach which we previously developed with the eye on the category of schemes (Lowen and Mestdagh, J. Pure Appl. Algebra 217(11), 2180-2197, 2013). We also show how our results for $C^{*}$-algebras can naturally be adapted to the opposite Woronowicz category of nondegenerate algebras over a commutative ring.
\end{abstract}

Keywords Functional topology $\cdot \mathrm{C}^{*}$-algebra $\cdot$ Woronowicz category $\cdot$ Proper morphism

To Horst Herrlich: through hugely inspiring mathematics and moonlit memories your wonderful mind shines on.

The authors acknowledge the support of the European Union for the ERC grant No 257004-HHNcdMir and the support of the Research Foundation Flanders (FWO) under Grant No G.0112.13N.

\section{Wendy Lowen}

wendy.lowen@uantwerpen.be

Joris Mestdagh

mestdaghjoris@hotmail.com

1 Departement Wiskunde-Informatica, Middelheimcampus, Middelheimlaan 1, 2020 Antwerp, Belgium 


\section{Introduction}

By the famous Gelfand-Naimark theorem, the category of locally compact Hausdorff spaces with proper maps is dual to the category of commutative $C^{*}$-algebras with $C^{*}$-morphisms. This theorem forms the basis for the point of view of noncommutative geometry, where noncommutative $C^{*}$-algebras are considered as "noncommutative spaces". An obvious shortcoming in the above result is the a priori restriction to proper maps between topological spaces. This problem is remedied by turning to the Woronowicz category of $C^{*}$-algebras $\operatorname{Wor}\left(C^{*}\right.$-Alg), of which the restriction to commutative $C^{*}$-algebras is equivalent to the category of locally compact Hausdorff spaces with all continuous maps. The definition of the Woronowicz category is somewhat involved, and makes essential use of the construction of the multiplier algebra, which is the largest unitization of a $C^{*}$-algebra and constitutes the noncommutative counterpart of the Čech-Stone compactification. This suggests that unital $C^{*}$-algebras should somehow be considered as compact objects.

The main aim of this paper is to introduce and investigate notions of compactness and properness for $C^{*}$-algebras in the context of so-called functional topology on the category $\operatorname{Wor}\left(C^{*}-\mathbf{A l g}\right)^{\text {op }}$. In very general terms, functional topology is an approach to developing topological concepts in a category based upon certain (axiomatically defined) classes of maps. This basic idea dates back at least to the seventies with the work of Herrlich [10], Manes [18], Penon [20, 21], and in the presence of a factorization system it led to a richer theory in the work of Herrlich, Salicrup and Strecker [12], applicable in topology, group theory and order. In the 90 's, on categories endowed with closure operators, a close resemblance with the topological situation was obtained by Clementino, Giuli and Tholen [4], applicable to Birkhoff closure spaces, uniform spaces, topological groups and locales. The idea of using a class of closed morphisms in order to derive both proper and separated morphisms from it was contained in Tholen's [22], and gave rise to the theory of functional topology as developed by Clementino, Giuli and Tholen in the presence of a factorization system [5], which is applicable for instance to approach spaces as demonstrated by Colebunders, Lowen and Wuyts [6]. An approach by Hofmann and Tholen later focussed the attention on the proper maps as primary, and is applicable to general categories of lax algebras [13].

In [16], after noticing the similarities between functional topology and the classes of proper and separated morphisms of schemes introduced by Grothendieck, the authors extended the framework from [5], making it somewhat more flexible without greatly diminishing its power, and in particular making it applicable to schemes [16]. Surprisingly, the resulting framework turns out to be well-suited to incorporate the Woronowicz category. The main new element we need is a shift from finitely complete categories to appropriate monoidal categories. Indeed the category Wor $\left(C^{*}-\mathbf{A l g}\right)^{\text {op }}$ has no (known) binary products but is endowed with a monoidal structure given by the maximal tensor product. In the classical functional topology setup, a proper class of morphisms is a class containing the isomorphisms, which is closed under compositions and pullback-stable. This last property does not seem quite natural in a monoidal context. To find the appropriate replacement, we investigate generalized limits in a more general framework: that of categories endowed with a relation $R$, that is a collection of compatible relations on the classes of morphisms with common domain. The notion of a relation on a category is dual to the notion of a cover relation in the sense of Janelidze [14], which we call a co-relation in the present paper. Associated $R$-limits originate from considering cones in which some of the involved morphisms are required to be in relation. For instance, we define the $R$-pullback of two morphisms 
$f: A \longrightarrow C$ and $g: B \longrightarrow C$ to consist of a universal object $P$ with morphisms $p_{1}: P \longrightarrow A$ and $p_{2}: P \longrightarrow B$ such that $f p_{1}=g p_{2}$ and $\left(p_{1}, p_{2}\right) \in R$. Natural notions of $R$-commutative objects and $R$-central morphisms relative to a relation can be defined and expressed in terms of generalized limits. After establishing some basic results involving $R$ limits in Section 4, in Section 5 we develop some approaches to functional topology in a category with $R$-pullbacks and a final object. We will refer to these approaches as "tensor functional topology".

- $\quad$ The most basic approach takes an $R$-proper class $\mathbb{F}$ of proper morphisms as input. With respect to $\mathbb{F}$ one defines compact objects as objects for which the unique morphism to the final object is proper, separated morphisms as morphisms with a commutative domain and proper diagonal, and Hausdorff objects as commutative objects with proper diagonal. Starting the theory from an $R$-proper class rather than a closed class is preferable as for general $R$, we cannot naturally associate an $R$-proper class to a closed class due to the weaker composability properties of $R$-pullback diagrams.

- If $\mathcal{C}$ is endowed with a factorization system $(\mathbb{E}, \mathbb{M})$, one can obtain a closed class $\mathbb{F}$ of closed morphisms from a so-called $(\mathbb{E}, \mathbb{M})$-closed structure $\left(\mathbb{P}, \mathbb{F}_{0}\right)$ consisting of an auxiliary $R$-proper class $\mathbb{F}_{0}$ of closed immersions and an auxiliary closed class $\mathbb{P}$ of surjections, following [16]. I n general, $\mathbb{F}$ will not be $R$-proper. In case of general $R$, we may additionally require $\mathbb{F}$ to be an $R$-proper class, in which case we speak of an $(\mathbb{E}, \mathbb{M})$-proper structure with proper morphisms $\mathbb{F}$.

Many classical results from functional topology still hold true in tensor functional topology, although sometimes additional centrality conditions need to be imposed. To understand this, it is worth noting that if we restrict our category to the full subcategory of commutative objects, the relation $R$ restricts to the standard relation for which any two morphisms with common domain are related.

In Section 6, we give an account of tensor functional topology in the opposite Woronowicz category Wor $\left(C^{*} \text {-Alg }\right)^{\text {op }}$. The necessary background on this category is recalled in Section 2. For a morphism $f: A \longrightarrow B$ in Wor $\left(C^{*} \text {-Alg }\right)^{\text {op }}$, we write $F: B \longrightarrow \mathcal{M}(A)$ for the associated Woronowicz-morphism. Two morphisms $f: A \longrightarrow B$ and $f^{\prime}: A \longrightarrow B^{\prime}$ are $R$-related if the associated Woronowicz-morphisms $F$ and $F^{\prime}: B^{\prime} \longrightarrow \mathcal{M}(A)$ commute. We make the following definitions for $f: A \longrightarrow B$ :

- $f$ is in $\mathbb{F}$ if $F(B) \subseteq A$;

- $f$ is in $\mathbb{M}$ if $F(B)=A$;

- $f$ is in $\mathbb{E}$ if $F$ is an isometry.

We show that $\mathbb{F}$ is an $R$-proper class, for which compact objects correspond precisely to unital algebras, and all commutative algebras are Hausdorff. Further, $(\mathbb{E}, \mathbb{M})$ is a factorization system on $\operatorname{Wor}\left(C^{*} \text {-Alg }\right)^{\text {op }}$ and $(\mathbb{E} \cap \mathbb{F}, \mathbb{M})$ is an $(\mathbb{E}, \mathbb{M})$-proper structure with $\mathbb{F}$ as its class of proper morphisms. Restricting $\operatorname{Wor}\left(C^{*} \text {-Alg }\right)^{\text {op }}$ to the commutative objects yields a category equivalent to locally compact Hausdorff spaces with continuous maps. We show that the restriction of $(\mathbb{E}, \mathbb{M})$ to this category corresponds precisely to the factorization system of dense maps and closed embeddings. Further, the class $\mathbb{F}$ restricts precisely to the proper continuous maps.

The application of tensor functional topology to $C^{*}$-algebras raises the natural question how much of this approach goes through for ordinary algebras. As we recall in Section 3, a multiplier algebra is known to exist for nondegenerate $k$-algebras over a commutative 
ring $k$. We further introduce the nondegenerate tensor product between nondegenerate $k$-algebras. We obtain a Woronowicz category Wor $(k$-Alg) of nondegenerate $k$-algebras which closely resembles the category $\operatorname{Wor}\left(C^{*}\right.$-Alg). In particular, its opposite category can be endowed with the commutation relation $R$ and classes of morphisms $\mathbb{F}, \mathbb{M}, \mathbb{E}$ analogous to the higher classes. All higher statements about the $C^{*}$-algebra case have precise parallels for nondegenerate $k$-algebras, as shown in Section 6 . In fact, under mild assumptions on a symmetric monoidal closed category $\mathcal{C}$, a Woronowicz category of nondegenerate semigroup objects in $\mathcal{C}$ can be defined, and Wor $(k-\mathbf{A l g})$ can be obtained from this general construction by taking $\mathcal{C}$ to be the category of $k$-modules [17]. The development of tensor functional topology in these more general Woronowicz categories is work in progress.

\section{The Woronowicz Category of $C^{*}$-Algebras}

The construction of the Woronowicz category for $C^{*}$-algebras originates with Woronowicz [24]. We recall the details for convenience and to fix notation. More details and proofs can be found in Wegge-Olsen [23] and Lance [15]. We further prove the existence and construction of co-equalizers in the Woronowicz category (Theorem 2.14).

Definition 2.1 A $C^{*}$-algebra is a (possibly noncommutative, possibly nonunital) $\mathbb{C}$-algebra equipped with a norm $\|-\|$ and an involution $(-)^{*}: A \longrightarrow A$ such that

(1) $(\lambda x+\mu y)^{*}=\bar{\lambda} x^{*}+\bar{\mu} y^{*}$ for each $\lambda, \mu \in \mathbb{C}$ and $x, y \in A$.

(2) $(x y)^{*}=y^{*} x^{*}$ for each $x, y \in A$.

(3) $x^{* *}=x$ for each $x \in A$.

(4) $A$ is complete with respect to the norm \|\| .

(5) $\|x y\| \leq\|x\|\|y\|$ for each $x, y \in A$.

(6) $\left\|x x^{*}\right\|=\|x\|^{2}$ for each $x \in A$.

A $C^{*}$-algebra is called unital if it is unital as an algebra, i.e. if there is a unit element $1_{A} \in A$ such that $1_{A} a=a=a 1_{A}$ for each $a \in A$.

The $C^{*}$-morphisms are defined as follows:

Definition 2.2 Let $A$ and $B$ be $C^{*}$-algebras and let $f: A \longrightarrow B$ be a function. We say that $f$ is a $C^{*}$-morphism if

(1) $f$ is linear,

(2) $f(x y)=f(x) f(y)$ for each $x, y \in A$,

(3) $f\left(x^{*}\right)=f(x)^{*}$ for each $x \in A$.

If $A$ and $B$ are unital, then we say that $f$ is unital if $f\left(1_{A}\right)=1_{B}$.

One can show that each $C^{*}$-morphism $f$ is continuous and has norm $\|f\| \leq 1$. This choice of morphisms makes the $C^{*}$-algebras into a category, which we will call $C^{*}$-Alg. The theorem of Gelfand-Naimark classifies the commutative $C^{*}$-algebras. For a locally compact Hausdorff space $X$, we obtain the commutative $C^{*}$-algebra $\mathcal{C}_{0}(X, \mathbb{C})$ of continuous functions from $X$ to $\mathbb{C}$ which vanish at infinity. For a compact Hausdorff space $X$, we obtain the unital commutative $C^{*}$-algebra $\mathcal{C}(X, \mathbb{C})$ of continuous functions from $X$ to $\mathbb{C}$. In this case, we have $\mathcal{C}(X, \mathbb{C})=\mathcal{C}_{0}(X, \mathbb{C})$. 
Theorem 2.3 (Gelfand-Naimark) [23] The category of locally compact Hausdorff spaces with proper maps as morphisms is equivalent to the category of commutative $C^{*}$-algebras with $C^{*}$-morphisms as morphisms. The (contravariant) equivalence is given by associating to each locally compact Hausdorff space $X$, the commutative $C^{*}$-algebra $\mathcal{C}_{0}(X, \mathbb{C})$. Furthermore, under this equivalence, the category of compact Hausdorff spaces with continuous maps as morphisms is equivalent to the category of unital commutative $C^{*}$-algebras with unital $C^{*}$-morphisms as morphisms.

While the proper continuous functions between locally compact Hausdorff spaces correspond to the $C^{*}$-morphisms under this equialence, the same is not true for all the continuous functions. To be able to model all continuous functions, we need the concept of the multiplier algebra.

Definition 2.4 Let $A$ be a $C^{*}$-algebra. We say that an ideal $J$ of $A$ is an essential ideal if every other nonzero ideal in $A$ has nonzero intersection with $A$. Or equivalently, when the annihilator $J^{\perp}=\{a \in A \mid a J=0\}$ is zero.

Definition 2.5 A unitization of a $C^{*}$-algebra $A$ is an embedding of $A$ into a unital $C^{*}$ algebra $B$ such that $A$ is an essential ideal of $B$.

Since unital $C^{*}$-algebras correspond to compact Hausdorff spaces in Theorem 2.3, we see that the concept of unitization corresponds to compactification. The largest compactification is of course the Čech-Stone compactification. Likewise, every $C^{*}$-algebra has a largest unitization, which we will call the multiplier algebra.

Theorem 2.6 [23] Let $A$ be a $C^{*}$-algebra. There exists a unique unitization $\mathcal{M}(A)$ of $A$ such that if $A$ is embedded as an ideal in a $C^{*}$-algebra $B$, then there exists a unique morphism $\mu: B \longrightarrow \mathcal{M}(A)$ such that $\mu$ restricts to the identity on A. Moreover, $\mu$ is injective if and only if $A$ is essential in $B$.

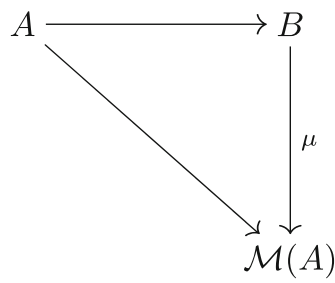

We call $\mathcal{M}(A)$ the multiplier algebra of $A$.

If $A=\mathcal{C}_{0}(X, \mathbb{C})$ for a locally compact Hausdorff space $X$, then the multiplier algebra $\mathcal{M}(A)$ is exactly $\mathcal{C}(\beta X, \mathbb{C}) \cong \mathcal{C}_{b}(X, \mathbb{C})$, the commutative $C^{*}$-algebra of bounded continuous functions from $X$ to $\mathbb{C}$, where $\beta X$ is the Čech-Stone compactification of $X$.

The multiplier algebra is not functorial. More precisely, if $f: A \longrightarrow B$ is a $C^{*}$-morphism, then this does not need to induce a morphism $\mathcal{M}(A) \longrightarrow \mathcal{M}(B)$.

Definition 2.7 Let $A$ be a $C^{*}$-algebra. Let $H$ be a Hilbert space such that $A$ is embedded as a subspace of $\mathcal{B}(H)$. The strict topology on $A$ is the locally convex topology generated by the seminorms $x \longrightarrow\|x a\|$ and $x \longrightarrow\|a x\|$ for $x \in \mathcal{B}(H)$ and $a \in A$.

One can prove that the multiplier algebra $\mathcal{M}(A)$ is the strict completion of $A$. 
For $C^{*}$-algebras $A$ and $B$, we call a $C^{*}$-morphism $F: A \longrightarrow \mathcal{M}(B)$ nondegenerate if $F(A) B$ is dense in $B$. We are now able to state the following extension theorem:

Theorem 2.8 [15] Let $A$ and $B$ be $C^{*}$-algebras and let $F: A \longrightarrow \mathcal{M}(B)$ be a $C^{*}$ morphism. The following conditions are equivalent:

(1) $F$ is nondegenerate.

(2) $F(A) B=B$.

(3) $B F(A)=B$.

(4) $F$ is the restriction to $A$ of a unique unital $C^{*}$-morphism $\mathcal{M}(A) \longrightarrow \mathcal{M}(B)$ which is strictly continuous on the unit ball.

A $C^{*}$-morphism $F: A \longrightarrow \mathcal{M}(B)$ that satisfies the conditions of the previous theorem will be called a Woronowicz-morphism from $A$ to $B$. The unique unital extension of $F$ : $A \longrightarrow \mathcal{M}(B)$ from (4) will be denoted by $\bar{F}: \mathcal{M}(A) \longrightarrow \mathcal{M}(B)$.

The continuous maps between locally compact Hausdorff spaces $X$ and $Y$ correspond exactly to the Woronowicz-morphisms from $\mathcal{C}_{0}(Y, \mathbb{C})$ to $\mathcal{C}_{0}(X, \mathbb{C})$. With this in mind, we define the category $\operatorname{Wor}\left(C^{*}\right.$-Alg $)$ to be the category of all $C^{*}$-algebras with Woronowiczmorphisms. Note that the above theorem says that we can compose such morphisms, thus the Woronowicz-category is well defined. Naturally, we are more interested in the opposite category Wor $\left(C^{*} \text {-Alg }\right)^{\text {op }}$ since this category can be seen as a category of "noncommutative topological spaces."

Recall that the multiplier algebra was not functorial with respect to the $C^{*}$-morphisms. This is solved by the Woronowicz-morphisms: any Woronowicz-morphism from $A$ to $B$ gives rise to a unique unital $C^{*}$-morphism $\mathcal{M}(A) \longrightarrow \mathcal{M}(B)$.

We would like to form tensor products of $C^{*}$-algebras $A$ and $B$. An obvious definition is to form the algebraic tensor product $A \otimes B$ and to complete this with respect to a suitable norm. A suitable norm should satisfy $\|x \otimes y\| \leq\|x\|\|y\|$ (this is called a subcross norm). The largest possible subcross norm is called the maximal norm:

Definition 2.9 Let $A$ and $B$ be $C^{*}$-algebras and define for each $t \in A \otimes B$

$$
\mu(t)=\sup \left\{\alpha(t) \mid \alpha \text { is a } C^{*} \text {-seminorm on } A \otimes B\right\} .
$$

This is called the maximal $C^{*}$-norm on $A \otimes B$. The completion with respect to this norm is denoted by $A \otimes_{\mu} B$.

Definition 2.10 Let $A, B$ and $C$ be (possibly noncommutative, possibly nonunital) rings and let $f: A \longrightarrow C$ and $g: B \longrightarrow C$ be ring-morphisms. We say that $f$ and $g$ commute if for each $a \in A$ and $b \in B$ we have that $f(a) g(b)=g(b) f(a)$.

Tensor products do not form a coproduct for $C^{*}$-algebras, but we do have the following theorem:

Theorem 2.11 [23] Let $f_{k}: A_{k} \longrightarrow B_{k}, k=1,2$ be morphisms of $C^{*}$-algebras, then $f_{1} \otimes f_{2}$ extends by continuity to a morphism $f_{1} \otimes_{\mu} f_{2}: A_{1} \otimes_{\mu} A_{2} \longrightarrow B_{1} \otimes_{\mu} B_{2}$.

If $g_{k}: A_{k} \longrightarrow C, k=1,2$ are commuting morphisms of $C^{*}$-algebras, then $g_{1} \otimes g_{2}$ extends by continuity to a morphism $g_{1} \otimes_{\mu} g_{2}: A_{1} \otimes_{\mu} A_{2} \longrightarrow C$. 
We collect some facts about the category $\operatorname{Wor}\left(C^{*}\right.$-Alg):

Proposition 2.12 If $F: A \longrightarrow \mathcal{M}(B)$ is a Woronowicz-morphism from $A$ to $B$ which constitutes an isomorphism in $\operatorname{Wor}\left(C^{*}-\operatorname{Alg}\right)$, then $F(A)=B$.

Proof Let $G: B \longrightarrow \mathcal{M}(A)$ be the inverse of $F$. Take an $a \in A$, then we have by nondegeneracy of $G$ that there exist $b_{i} \in B$ and $a_{i} \in A$ such that $a=\sum G\left(b_{i}\right) a_{i}$. But then $F(a)=\sum b_{i} F\left(a_{i}\right)$. But since $B$ is an ideal of $\mathcal{M}(B)$, this implies that $F(a) \in B$. This shows that $F(A) \subseteq B$. Likewise, we have that $G(B) \subseteq A$. Now take $b \in B$, then $b=F(G(b))$, and thus $b \in F(A)$.

Proposition 2.13 [23] Any surjection of $C^{*}$-algebras $p: A \longrightarrow B$ induces a nondegenerate morphism $P: A \longrightarrow \mathcal{M}(B)$.

Theorem 2.14 The co-equalizer in the category Wor $\left(C^{*}\right.$-Alg) exists. In particular, the coequalizer of two nondegenerate $C^{*}$-morphisms $F, G: A \longrightarrow \mathcal{M}(B)$ is given by $B / J$, where $J$ is the two-sided ideal in $B$ generated by $F(a) b-G(a) b$ and $b F(a)-b G(a)$ for each $a \in A$ and $b \in B$.

Proof Let $p: B \longrightarrow B / J$ be the canonical surjection. This surjection extends to a nondegerate morphism $P: B \longrightarrow \mathcal{M}(B / J)$. We prove that $\bar{P} F=\bar{P} G$. Let $\left(b_{i}\right)_{i \in I}$ be an approximate unit in $B$, i.e. it is a net in $B$ which converges strictly to the unit in $\mathcal{M}(B)$. By strict continuity, we know that $\left(\bar{P}\left(b_{i}\right)\right)_{i \in I}$ converges to the unit in $\mathcal{M}(B / J)$ (see [23]). Thus follows that $F(a) b_{i}$ converges strictly to $F(a)$ and that $G(a) b_{i}$ converges strictly to $G(a)$. By definition of $C$ as a quotient space, we have that $\bar{P}\left(F(a) b_{i}\right)=\bar{P}\left(G(a) b_{i}\right)$ and since $\bar{P}\left(F(a) b_{i}\right)$ converges to $\bar{P}(F(a))$ and $\bar{P}\left(G(a) b_{i}\right)$ converges to $\bar{P}(G(a))$, we have that $\bar{P}(G(a))=\bar{P}(G(a))$.

Let $H: \mathcal{B} \longrightarrow \mathcal{M}(C)$ be a Woronowicz morphism from $B$ to $C$ such that $\bar{H} F=\bar{H} G$. Then $H(J)=0$ and thus there is a unique morphism $K: B / J \longrightarrow \mathcal{M}(C)$ such that $H=\bar{K} P$. This is clearly nondegenerate since $H$ is.

Proposition 2.15 Let $G: A \longrightarrow \mathcal{M}(C)$ and $G: B \longrightarrow \mathcal{M}(C)$ be two Woronowiczmorphisms. If $F$ and $G$ commute, then so do their extensions $\bar{F}$ and $\bar{G}$.

Proof Take $x \in \mathcal{M}(A)$ and $y \in \mathcal{M}(B)$. Then there exist nets $\left(a_{i}\right)_{i \in I}$ in $A$ and $\left(b_{j}\right)_{j \in J}$ in $B$ such that $a_{i} \longrightarrow x$ strictly and $b_{i} \longrightarrow y$ strictly. But then $F\left(a_{i}\right)$ converges strictly to $\bar{F}(x)$ and $G\left(b_{j}\right)$ converges strictly to $\bar{G}(y)$. Then $F\left(a_{i}\right) G\left(b_{j}\right)$ converges strictly to $\bar{F}(x) \bar{G}(y)$ and $G\left(b_{j}\right) F\left(a_{i}\right)$ converges strictly to $\bar{G}(y) \bar{F}(x)$. Since $F\left(a_{i}\right) G\left(b_{j}\right)=G\left(b_{j}\right) F\left(a_{i}\right)$, it follows that $\bar{F}(x) \bar{G}(y)=\bar{G}(y) \bar{F}(x)$.

Proposition 2.16 [15] Let $M: A \longrightarrow \mathcal{M}(C)$ be an injective and nondegenerate $C^{*}$ morphism. Then $M$ extends uniquely to an isometric, unital $C^{*}$-morphism $\bar{M}: \mathcal{M}(A) \longrightarrow$ $\mathcal{M}(C)$. Furthermore, the image of $\bar{M}$ is given by the idealizer of $M(A)$, i.e.

$$
\bar{M}(\mathcal{M}(A))=\{x \in \mathcal{M}(C) \mid x M(A) \subseteq M(A) \text { and } M(A) x \subseteq M(A)\} .
$$




\section{The Woronowicz Category of Associative Algebras}

In this section we generalize the Woronowicz category to nondegenerate associative algebras over a commutative ground ring $k$ with unit. The basic notions necessary for this generalization can be found in De Commer, Van Daele [7]. In [17], we show that under mild assumptions on a symmetric monoidal closed category $\mathcal{C}$, a Woronowicz category of nondegenerate semigroup objects in $\mathcal{C}$ can be defined. The Woronowicz category of this section can be obtained from this general construction by taking $\mathcal{C}$ to be the category of $k$-modules. However, the general categorical proofs turn out to be much more involved. In this section we provide a self-contained treatment of the results for associative $k$-algebras.

Definition 3.1 A $k$-algebra $A$ is nondegenerate if the following hold for all $a \in A$ :

(1) If $a b=0$ for each $b \in A$, then $a=0$.

(2) If $b a=0$ for each $b \in A$, then $a=0$.

(3) We have that $A=A A:=\left\{\sum_{i} a_{i} a_{i}^{\prime} \mid a_{i}, a_{i}^{\prime} \in A\right\}$.

The category of all nondegenerate $k$-algebras with the usual $k$-algebra morphisms is denoted by $k-$ Alg.

Definition 3.2 The multiplier algebra of a nondegenerate $k$-algebra $A$ is the algebra $\mathcal{M}(A)$ consisting of couples $(\lambda, \rho)$, where $\lambda$ and $\rho$ are maps $A \longrightarrow A$ such that

$$
\rho(a) b=a \lambda(b) \text { for all } a, b \in A .
$$

This $k$-algebra is a $k$-unital algebra equipped with the following operations:

(1) For all $(\lambda, \rho),\left(\lambda^{\prime}, \rho^{\prime}\right) \in \mathcal{M}(A)$ and $r, s \in A$, we set

$$
r(\lambda, \rho)+s\left(\lambda^{\prime}, \rho^{\prime}\right)=\left(r \lambda+s \lambda^{\prime}, r \rho+s \rho^{\prime}\right) .
$$

(2) For all $(\rho, \lambda),\left(\rho^{\prime}, \lambda^{\prime}\right) \in \mathcal{M}(A)$, we set

$$
(\lambda, \rho) \cdot\left(\lambda^{\prime}, \rho^{\prime}\right)=\left(\lambda \circ \lambda^{\prime}, \rho^{\prime} \circ \rho\right) .
$$

(3) The unit is given by $\left(\operatorname{Id}_{A}, \operatorname{Id}_{A}\right)$.

Lemma 3.3 If $A$ is a nondegenerate $k$-algebra and if $(\lambda, \rho) \in \mathcal{M}(A)$, then both $\lambda$ and $\rho$ are $k$-linear. Furthermore, it holds for each $a, b \in A$ that

$$
\lambda(a b)=\lambda(a) b \text { and } \rho(a b)=a \rho(b) .
$$

Proof Let $a, b, c \in A$ be arbitrary, and let $r, s \in k$, then

$$
\begin{aligned}
c \lambda(r a+s b) & =\rho(c)(r a+s b) \\
& =r \rho(c) a+s \rho(c) b \\
& =c(r \lambda(a)+s \lambda(b))
\end{aligned}
$$

Since $c$ is arbitrary and $A$ is nondegenerate, it follows that $\lambda(r a+s b)=r \lambda(a)+s \lambda(b)$. The other statements in the lemma have a similar proof.

There is a natural $k$-algebra morphism $\iota: A \longrightarrow \mathcal{M}(A): a \longrightarrow\left(\lambda_{a}, \rho_{a}\right)$ where

$$
\lambda_{a}(b)=a b \text { and } \rho_{a}(b)=b a .
$$


Lemma 3.4 Let $A$ be a nondegenerate k-algebra. Then the map $\iota: A \longrightarrow \mathcal{M}(A)$ is injective.

Proof Assume that for an $a \in A$, we have that $\iota(a)=0$. This means that $\lambda_{a}=0$ and $\rho_{a}=0$. Hence for each $b \in A$, we have that $a b=0$ and $b a=0$. The nondegeneracy of $A$ then implies that $a=0$. Thus $\iota$ is injective.

Lemma 3.5 Let $A$ be a nondegenerate $k$-algebra, then we have for each $(\rho, \lambda) \in \mathcal{M}(A)$ that

$$
(\lambda, \rho) \cdot \iota(a)=\iota(\lambda(a)) \text { and } \iota(a) \cdot(\lambda, \rho)=\iota(\rho(a)) .
$$

Thus $\iota(A)$ is an ideal of $\mathcal{M}(A)$. Hence if $A$ is unital, then $\iota$ is an isomorphism.

Proof We have that

$$
(\lambda, \rho) \cdot \iota(a)=\left(\lambda \lambda_{a}, \rho_{a} \rho\right)
$$

For $b \in A$, we have that

$$
\left(\lambda \lambda_{a}\right)(b)=\lambda(a b)=\lambda(a) b .
$$

Thus $\lambda \lambda_{a}=\lambda_{\lambda(a)}$. We also have

$$
\left(\rho_{a} \rho\right)(b)=\rho(b) a=a \lambda(b) .
$$

Thus $\rho_{a} \rho=\rho_{\lambda(b)}$. The rest of the lemma is similar.

Corollary 3.6 Let $A$ be a nondegenerate k-algebra, and let $(\lambda, \rho) \in \mathcal{M}(A)$. If $(\lambda, \rho)$. $\iota(a)=0$ for each $a \in A$, then $(\lambda, \rho)=0$.

In what follows, we will usually not explicitly write $\iota$. Thus we view $A$ as a subset of $\mathcal{M}(A)$.

Definition 3.7 Let $A$ and $B$ be nondegenerate $k$-algebras. We say that an algebra morphism $F: A \longrightarrow M(B)$ is nondegenerate if $B=F(A) B$ and $B=B F(A)$. A Woronowiczmorphism between $A$ and $B$ is then defined as a nondegenerate algebra morphism $F$ : $A \longrightarrow \mathcal{M}(B)$.

Proposition 3.8 A Woronowicz-morphism $F: A \longrightarrow \mathcal{M}(B)$ can be extended to a unique unital algebra morphism $\bar{F}: \mathcal{M}(A) \longrightarrow \mathcal{M}(B)$.

Proof Let $(\lambda, \rho) \in \mathcal{M}(A)$. Every $b \in B$ can be written as $b=\sum_{i} F\left(a_{i}\right) b_{i}$. Now we define $\lambda^{\prime}(b)=\sum F\left(\lambda\left(a_{i}\right)\right) b_{i}$. Using nondegeneracy of $A$ and $F$, one easily checks that this is well-defined. Similarly, one defines $\rho^{\prime}(b)=\sum b_{i} F\left(\rho\left(a_{i}\right)\right)$. The required extension is $\bar{F}: \mathcal{M}(A) \longrightarrow \mathcal{M}(B):(\lambda, \rho) \longrightarrow\left(\lambda^{\prime}, \rho^{\prime}\right)$.

Assume that $\widehat{F}: \mathcal{M}(A) \longrightarrow \mathcal{M}(B)$ is another extension of $F$. Then we have for each $x \in \mathcal{M}(A)$ and $b^{\prime} F\left(a^{\prime}\right) \in B$ that $a^{\prime} x \in A$. Hence

$$
b^{\prime} F\left(a^{\prime}\right)(\bar{F}(x)-\widehat{F}(x))=0
$$

Thus for each $b \in B$ we have that $b(\bar{F}(x)-\widehat{F}(x))=0$. It follows that $\bar{F}(x)=\widehat{F}(x)$. 
This proposition has as a corollary that we can compose nondegenerate maps. Indeed, if $F: A \longrightarrow \mathcal{M}(B)$ and $G: B \longrightarrow \mathcal{M}(C)$ are nondegenerate, then $G$ extends to a morphism $\bar{G}: \mathcal{M}(B) \longrightarrow \mathcal{M}(C)$, and the composition $\bar{G} F$ makes sense.

Lemma 3.9 The composition $\bar{G} F$ is nondegenerate.

Proof Take $c \in C$, then by nondegeneracy of $G$, we can write $c=\sum_{i} G\left(b_{i}\right) c_{i}$, where $b_{i} \in B$ and $c_{i} \in C$. Now, since $F$ is nondegenerate, we can write $b_{j}=\sum_{j} F\left(a_{i, j}\right) b_{i, j}$, where $a \in A$ and $b^{\prime} \in B$. Thus $c=\sum_{i, j} \bar{G}\left(F\left(a_{i, j}\right)\right) F\left(b_{i, j}\right) c_{i}$ and $G\left(b_{i, j}\right) c_{i} \in C$. Thus we have shown that $\bar{G}(F(A)) C=C$. Similarly, it follows that $C \bar{G}(F(A))=C$.

We can now form the category of all $k$-algebras with Woronowicz morphisms. We will denote this category as

\section{$\operatorname{Wor}(k-\mathbf{A l g})$}

Proposition 3.10 Let $F: A \longrightarrow \mathcal{M}(B)$ be an injective, nondegenerate map, then its extension $\bar{F}: \mathcal{M}(A) \longrightarrow \mathcal{M}(B)$ is injective too.

Proof Assume that $x \in \mathcal{M}(A)$ satisfies $\bar{F}(x)=0$. Then for each $a \in A$, we have that $F(a x)=F(a) \bar{F}(x)=0$. Since $f$ is injective on $A$ and since $a x \in A$, it follows that $a x=0$. This holds for each $a \in A$; it follows that $x=0$.

Proposition 3.11 For every surjective algebra morphism $g: A \longrightarrow B$, we have that $G:=$ $\iota g: A \longrightarrow \mathcal{M}(B)$ is nondegenerate and that this extends to a morphism $\bar{G}: \mathcal{M}(A) \longrightarrow$ $\mathcal{M}(B)$.

Proof Take $b \in B$, then there exists an $a \in A$ such that $g(a)=b$. We can write $a=$ $\sum_{i} a_{i}^{\prime} a_{i}^{\prime \prime}$. Hence $b=\sum_{i} g\left(a_{i}^{\prime}\right) g\left(a_{i}^{\prime \prime}\right)$. Since $g(A) \subseteq B$, it follows that $g\left(a_{i}^{\prime}\right), g\left(a_{i}^{\prime \prime}\right) \in B$. Hence $b \in g(A) B$ and $b \in B g(A)$.

Proposition 3.12 If $F: A \longrightarrow \mathcal{M}(B)$ is a Woronowicz-morphism which constitutes an isomorphism in Wor $(k$-Alg), then $F(A)=B$.

Proof Let $G: B \longrightarrow \mathcal{M}(A)$ be the inverse of $f$. Take an $a \in A$; then we have by nondegeneracy of $G$ that there exists $b_{i} \in B$ and an $a_{i} \in A$ such that $a=\sum G\left(b_{i}\right) a_{i}$. But then $F(a)=b_{i} F\left(a_{i}\right)$. But since $B$ is an ideal of $\mathcal{M}(B)$, this implies that $F(a) \in B$. This shows that $F(A) \subseteq B$. Likewise, we have that $G(B) \subseteq A$. Now take $b \in B$, then $b=\bar{F}(G(b))$, and thus $b \in F(A)$. 
The quotients of nondegenerate algebras do not need to be nondegenerate. But we do have the following

Proposition 3.13 Let $A$ be a nondegenerate algebra and let $I$ be an ideal of $A$. We let $\widehat{I}=\{a \in A \mid a b \in I$ and $b a \in I$ for all $b \in B\}$. Then

(1) $\widehat{I}$ is an ideal and $A / \widehat{I}$ is nondegenerate.

(2) If $F: A \longrightarrow \mathcal{M}(B)$ is a nondegenerate morphism between nondegenerate algebras such that $F(I)=0$. Then it follows that $F(\widehat{I})=0$ and $F$ induces a nondegenerate morphism $A / \widehat{I} \longrightarrow \mathcal{M}(B)$.

Proof (1) That $\widehat{I}$ is an ideal is easily checked. Assume that $p(x) p(y)=0$ for all $y \in A$. Then holds that $x y \in \widehat{I}$ for all $y \in A$. It follows that for each $y^{\prime} \in A$ that $x y y^{\prime} \in I$. But since $A$ is nondegenerate, we know that every element of $A$ has the form $\sum_{i} y_{i} y_{i}^{\prime}$. So for every $a \in A$, we have that $x a \in I$. Thus follows that $x \in \widehat{I}$ and thus $p(x)=0$.

Since $A=A A$, it follows at once that $A / J=(A / J)(A / J)$.

(2) Take $x \in \widehat{I}$. Then holds for each $a \in A$ that $a x \in I$ and $x a \in I$. Take $b \in B$, then by nondegeneracy, we can write $b=\sum_{i} F\left(a_{i}\right) b_{i}$. But then

$$
F(x) b=\sum_{i} F(x) F\left(a_{i}\right) b_{i}=\sum_{i} F\left(x a_{i}\right) b_{i}=0 .
$$

It follows that $F(x)=0$. The remainder of the proof is now clear.

Theorem 3.14 The co-equalizer in the category Wor( $k$-Alg) exists. In particular, the coequalizer of two nondegenerate morphisms $F, G: A \longrightarrow \mathcal{M}(B)$ is given by $B / \widehat{I}$, where $I$ is the two-sided ideal in $B$ generated by $F(a) b-G(a) b$ and $b F(a)-b G(a)$ for each $a \in A$ and $b \in B$.

Proof Since $B$ is nondegenerate, it follows that $B / \widehat{I}$ is nondegenerate. Let $p: B \longrightarrow$ $B / \widehat{I}$ be the canonical surjection. This surjection is nondegenerate and thus extends to a nondegenerate surjection $\bar{P}: \mathcal{M}(B) \longrightarrow \mathcal{M}(B / \widehat{I})$. We prove that $\bar{P} F=\bar{P} G$. Indeed, take $P(b) \in B / \widehat{I}$, then

$$
[\bar{P}(F(a))-\bar{P}(G(b))](\bar{P}(b))=\bar{P}(F(a) b-G(a) b)=0 .
$$

Hence $\bar{P}(F(a))=\bar{P}(G(a))$ for each $a \in A$.

Let $H: B \longrightarrow \mathcal{M}(C)$ be a nondegenerate morphism such that $\bar{H} F=\bar{H} G$. Then $H(\widehat{I})=0$ and thus there is a unique morphism $K: B / \widehat{I} \longrightarrow \mathcal{M}(C)$ such that $H=\bar{K} P$. This is clearly nondegenerate since $H$ is.

Proposition 3.15 Let $F: A \longrightarrow \mathcal{M}(C)$ and $G: B \longrightarrow \mathcal{M}(C)$ be two Woronowiczmorphisms. If $F$ and $G$ commute, so do their extensions $\bar{F}$ and $\bar{G}$. 
Proof Take $x \in \mathcal{M}(A)$ and $y \in B$. Take $c \in C$, then we can write $c=\sum_{i} c_{i} F\left(a_{i}\right)$. Note that $a_{i} x \in A$. Then

$$
\begin{aligned}
c \bar{F}(x) G(y) & =\sum_{i} c_{i} F\left(a_{i} x\right) G(y) \\
& =\sum_{i} c_{i} G(y) F\left(a_{i} x\right) \\
& =\sum_{i} c_{i} G(y) F\left(a_{i}\right) \bar{F}(x) \\
& =\sum_{i} c_{i} F\left(a_{i}\right) F(y) \bar{F}(x) \\
& =c G(y) \bar{F}(x) .
\end{aligned}
$$

It follows that $\bar{F}(x) G(y)=G(y) \bar{F}(x)$. Now let $x \in \mathcal{M}(A)$ and $y \in \mathcal{M}(B)$. Similarly, iIt follows that $\bar{F}(x) \bar{G}(y)=\bar{G}(y) \bar{F}(x)$.

Definition 3.16 For an associative $k$-algebra $A$ and for an ideal $I$ of $A$, we say that $I$ is nondegenerate if for each $x \in A$ the following hold:

(1) If for each $y \in A$ we have that $y x \in I$, then $x \in I$.

(2) If for each $y \in A$ we have that $x y \in I$, then $x \in I$.

The ideal $A$ is always nondegenerate in $A$. Furthermore, an algebra is nondegenerate if and only if $A=A A$ and $\{0\}$ is a nondegenerate ideal of $A$.

Proposition 3.17 The intersection of nondegenerate ideals is nondegenerate.

In particular, every ideal $I$ can be extended to a smallest nondegenerate ideal which contains it. We will denote this ideal by $\widetilde{I}$.

We give a more explicit description of $\{\widetilde{0}\}$.

Proposition 3.18 Let $A$ be an associative k-algebra. We have that $a \in\{\widetilde{0}\}$ if and only if there exists $n \geq 0$ such that for all $b_{1}, \ldots, b_{n} \in A$ we have $b_{1} b_{2} \ldots b_{n} a=0$ and $a b_{1} b_{2} \ldots b_{n}=$ 0 .

Proposition 3.19 If $A$ and $B$ are nondegenerate $k$-algebras, then $A \otimes B /\{\widetilde{0}\}$ is $a$ nondegenerate $k$-algebra.

For nondegenerate $k$-algebras $A$ and $B$, we put $A \widetilde{\otimes} B=A \otimes B /\{\widetilde{0}\}$ and we call it the nondegenerate tensor product.

Proposition 3.20 If $k$ is a field, then $A \widetilde{\otimes} B=A \otimes B$. 
Proof Assume for $a \otimes b$ we have that $a a^{\prime} \otimes b b^{\prime}=0$ for all $a^{\prime} \in A$ and $b^{\prime} \in B$. This implies that there exists finitely supported maps $\lambda_{a^{\prime}, b^{\prime}}: B \longrightarrow R$ such that

$$
b b^{\prime}=\sum_{f \in B} \lambda_{a^{\prime}, b^{\prime}}(f) f,
$$

and

$$
\lambda_{a^{\prime}, b^{\prime}}(f) a a^{\prime}=0 \text { for all } f \in B
$$

If $\lambda_{a^{\prime}, b^{\prime}}(f)=0$ for all $f \in B$, then follows that $b b^{\prime}=0$. Otherwise, it follows by taking inverses that $a a^{\prime}=0$.

Thus if there exists some $b^{\prime}$ such that $b b^{\prime} \neq 0$, then it must hold that $a a^{\prime}=0$ for all $a^{\prime} \in A$. Thus by nondegeneracy of $A$, we have that $a=0$ and thus $a \otimes b=0$. 0 .

Otherwise, it must hold that $b b^{\prime}=0$ for all $b^{\prime} \in B$. But then $b=0$ and hence $a \otimes b=$

Proposition 3.21 For nondegenerate algebras $A$ and $B$, there exist commuting, nondegenerate maps

$$
J_{A}: A \longrightarrow \mathcal{M}(A) \longrightarrow \mathcal{M}(A \widetilde{\otimes} B)
$$

and

$$
J_{B}: B \longrightarrow \mathcal{M}(B) \longrightarrow \mathcal{M}(A \widetilde{\otimes} B)
$$

Proof Take $(\lambda, \rho) \in \mathcal{M}(A)$, let $p: A \otimes B \longrightarrow A \widetilde{\otimes} B$ be the canonical quotient. Take $p(a \otimes b) \in A \widetilde{\otimes} B$, then we set $\lambda^{\prime}(p(a \otimes b))=p(\lambda(a) \otimes b)$ and $\rho^{\prime}(p(a \otimes b))=p(\rho(a) \otimes b)$. This is well-defined: if $a \otimes b \in\{0\}$ and if $I$ is any nondegenerate ideal of $A \otimes B$, then $a \otimes b \in I$. Thus $(x \otimes y)(\lambda(a) \otimes b)=(x \lambda(a)) \otimes(y b)=(\rho(x) a) \otimes(y b) \in I$. By nondegeneracy of $I$ follows that $\lambda(a) \otimes b \in I$. Since this is true for all nondegenerate ideals of $A \otimes B$, it follows that $\lambda(a) \otimes b \in\{0\}$. Similarly, we have that $\rho^{\prime}$ is well-defined.

So we have an element $\left(\lambda^{\prime}, \rho^{\prime}\right) \in \mathcal{M}(A \widetilde{\otimes} B)$. This shows that we have a morphism

$$
\mathcal{M}(A) \longrightarrow \mathcal{M}(A \otimes B):(\lambda, \rho) \longrightarrow\left(\lambda^{\prime}, \rho^{\prime}\right) .
$$

Similarly, we also have a morphism $\mathcal{M}(B) \longrightarrow \mathcal{M}(A \otimes B)$. These morphisms are clearly commuting. Note that for $\left(\lambda_{a}, \rho_{a}\right) \in \mathcal{M}(A)$, we have $\lambda_{a}^{\prime}\left(p\left(a^{\prime} \otimes b^{\prime}\right)\right)=p\left(a a^{\prime} \otimes b^{\prime}\right)$ and $\lambda_{a}^{\prime}\left(p\left(a^{\prime} \otimes b^{\prime}\right)\right)=p\left(a^{\prime} a \otimes b^{\prime}\right)$. We check nondegeneracy. Take $p(a \otimes b) \in A \widetilde{\otimes} B$. Then we can write $a=\sum_{i} a_{i} a_{i}^{\prime}$. But then

$$
p(a \otimes b)=\sum_{i} p\left(a_{i} a_{i}^{\prime} \otimes b\right)=\sum_{i} \lambda_{a_{i}}^{\prime}\left(p\left(a_{i}^{\prime} \otimes b\right) \in J_{A}(A)(A \widetilde{\otimes} B) .\right.
$$

\section{Generalized Colimits}

It is well known that in categories without (useful) coproducts, a monoidal structure may sometimes serve as an alternative. More generally, if a category is endowed with compatible relations $R_{C}$ on the sets of morphisms with common domain $C$, there results a natural notion of $R$-coproduct. The link between such relations on the one hand, and monoidal 
structures on the other hand, is studied by Janelidze [14]. In Section 4.1, we describe $R$ coproducts in the Woronowicz categories from the previous sections. In Section 4.2, we introduce more general $R$-colimits. Under an additional assumption on $R$, we prove some properties involving combinations of $R$-colimits. For instance, we show that in general $R$ pushouts cannot be composed, but they can be composed under an additional centrality requirement for one of the involved morphisms. In the sequel, we will make fundamental use of the dual notions, which are discussed in Section 4.3.

\subsection{Co-Relations and Generalized Coproducts}

Recall that for a category $\mathcal{C}$ and an object $C \in \mathcal{C}$, the slice category $\mathcal{C} / C$ is the category of all morphisms with codomain $C$.

Definition 4.1 [14] A co-relation $R$ on a category $\mathcal{C}$ consists of the datum of a relation $R_{C}$ on $\operatorname{Ob}(\mathcal{C} / C)$ for every object $C \in \mathcal{C}$, such that the following properties are satisfied:

(1) If $f: A \longrightarrow C$ and $g: B \longrightarrow C$ are $R_{C}$-related and if $h: D \longrightarrow A$ is an arbitrary morphism, then $f h$ and $g$ are $R_{C}$-related.

(2) If $f: A \longrightarrow C$ and $g: B \longrightarrow C$ are $R_{C}$-related and if $h: C \longrightarrow D$ is an arbitrary morphism, then $h f$ and $h g$ are $R_{D}$-related.

For simplicity, we usually denote $R_{C}$ simply by $R$ for all objects $C$. In [14], a co-relation $R$ on a category is called a cover relation and the author investigates cover relations associated to the natural order on subobjects (whence the terminology), as well as cover relations associated to monoidal structures. In this paper we are mainly interested in (generalizations of) the latter type, where in particular $R$ is symmetric. Note that in general, for a co-relation $R$ on $\mathcal{C}$, the opposite relations $R_{C}^{\mathrm{op}}$ for $C \in \mathcal{C}$ do not define a co-relation due to the asymmetric nature of condition (1). If they do define a co-relation, we denote it by $R^{\text {op }}$ and we call $R$ a bi-co-relation (bicover relation in [14]).

Example 4.2 (1) If $\mathcal{C}$ is an arbitrary category, then we can say that $f: A \longrightarrow C$ and $g: B \longrightarrow C$ are always $R$-related. We will call this co-relation standard.

(2) Let $F: A \longrightarrow \mathcal{M}(C)$ and $G: B \longrightarrow \mathcal{M}(C)$ be morphisms in Wor $\left(C^{*}\right.$-Alg). We say that $F$ and $G$ are $R$-related if they commute.

(3) Let Ring be the category of all (possibly noncommutative) unital rings with as morphisms the usual ring homomorphisms. Two morphisms $f$ and $g$ are $R$-related if they commute.

(4) Let $G: A \longrightarrow \mathcal{M}(C)$ and $G: B \longrightarrow \mathcal{M}(C)$ be in Wor( $k$-Alg). We say that $F$ and $G$ are $R$-related if they commute.

With these examples in mind, we make the following definition:

Definition 4.3 Let $\mathcal{C}$ be a category with a co-relation $R$ on $\mathcal{C}$.

(1) A morphism $f: B \longrightarrow A$ in $\mathcal{C}$ is $R$-central if $\left(f, 1_{A}\right) \in R$ and $R$-op-central if $\left(1_{A}, f\right) \in R$.

(2) An object $A \in \mathcal{C}$ is $R$-commutative if $\left(1_{A}, 1_{A}\right) \in R$.

The central morphisms in $\mathcal{C}$ constitute a left ideal in $\mathcal{C}$, that is if $f: A \longrightarrow B$ is central and $h: A^{\prime} \longrightarrow A$ is arbitrary, then $f h$ is central. The commutative objects in $\mathcal{C}$ form a 
full subcategory $\operatorname{Com}(\mathcal{C}) \subseteq \mathcal{C}$. The co-relation $R$ on $\mathcal{C}$ gives rise to an obvious restricted co-relation on any subcategory. In particular, the restricted co-relation on $\operatorname{Com}(\mathcal{C})$ is the standard relation on $\operatorname{Com}(\mathcal{C})$.

A co-relation $R$ on a category can be used to generalize the notions of coproduct and pushout:

Definition 4.4 Let $A$ and $B$ be objects of a category $\mathcal{C}$. The $R$-coproduct of $A$ and $B$ is an object $Q$ together with $R$-related morphisms $q_{A}: A \longrightarrow Q$ and $q_{B}: B \longrightarrow Q$ such that for every two $R$-related morphisms $f_{A}: A \longrightarrow C$ and $f_{B}: B \longrightarrow C$, there is a unique morphism $f: Q \longrightarrow C$ such that $f_{A}=f q_{A}$ and $f_{B}=f q_{B}$.

We denote the $R$-coproduct of $A$ and $B$ by $A \coprod^{R} B$. Clearly, if it exists, an $R$-coproduct is unique up to isomorphism. In [14], Janelidze characterizes the co-relations $R$ for which the associated $R$-coproduct defines a monoidal structure on $\mathcal{C}$. Note that unless $R$ is symmetric, we do not necessarily have $A \coprod^{R} B \cong B \coprod^{R} A$.

We can give a similar definition for the $R$-pushout:

Definition 4.5 Let $f: C \longrightarrow A$ and $g: C \longrightarrow B$ be morphisms in a category $\mathcal{C}$. The $R$ pushout of $f$ and $g$ is an object $Q$ together with $R$-related morphisms $q_{A}: A \longrightarrow Q$ and $q_{B}: B \longrightarrow Q$ such that $q_{A} f=q_{B} g$ and such that for every two $R$-related morphisms $f_{A}$ : $A \longrightarrow C$ and $f_{B}: B \longrightarrow C$ with $f_{A} f=f_{B} g$, there is a unique morphism $h: Q \longrightarrow C$ such that $f_{A}=h q_{A}$ and $f_{B}=h q_{B}$.

We denote the $R$-pushout of $f$ and $g$ as above by $A \coprod_{C}^{R} B$. Again, if it exists, an $R$ pushout is unique up to isomorphism. We call $q_{B}$ the $R$-pushout of $f$ by $g$, and by a pushout of $f$ we mean a pushout $q_{B}$ of $f$ by some morphism $g$.

If $R$ is the standard co-relation, then the $R$-coproduct and $R$-pushout are simply the usual coproduct and pushout.

Theorem 4.6 In the category Wor $\left(C^{*}-A l g\right), R$-coproducts exist and are given by the maximal tensor product.

Proof Let $A$ and $B$ be $C^{*}$-algebras. We can form the maximal tensor product $A \otimes_{\mu} B$. It is shown in [23] that

$$
A \otimes_{\mu} B \subseteq \mathcal{M}(A) \otimes_{\mu} \mathcal{M}(B) \subseteq \mathcal{M}\left(A \otimes_{\mu} B\right) .
$$

Thus the canonical maps $q_{A}: A \longrightarrow \mathcal{M}(A) \otimes_{\mu} \mathcal{M}(B): a \longrightarrow a \otimes_{\mu} 1$ and $q_{B}: B \longrightarrow$ $\mathcal{M}(A) \otimes_{\mu} \mathcal{M}(B): b \longrightarrow 1 \otimes_{\mu} b$ extend to maps $Q_{A}: A \longrightarrow \mathcal{M}\left(A \otimes_{\mu} B\right)$ and $Q_{B}:$ $B \longrightarrow \mathcal{M}\left(A \otimes_{\mu} B\right)$. These maps are clearly commuting. Furthermore, they are strictly continuous since

$$
\left\|q_{A}(a)\left(a^{\prime} \otimes_{\mu} b^{\prime}\right)\right\|=\left\|\left(a a^{\prime}\right) \otimes_{\mu} b^{\prime}\right\| \leq\left\|a a^{\prime}\right\|\left\|b^{\prime}\right\| .
$$

Now let $F_{A}: A \longrightarrow \mathcal{M}(C)$ and $F_{B}: B \longrightarrow \mathcal{M}(C)$ be commuting nondegenerate morphisms. Then we define $F: A \otimes B \longrightarrow \mathcal{M}(C)$ by $f(a \otimes b)=f_{A}(a) f_{B}(b)$. This is clearly the unique map for which $F_{A}=\bar{F} Q_{A}$ and $F_{B}=\bar{F} Q_{B}$. We must prove that $F$ is nondegenerate. For this, take $c \in C$. Then we can write $c=\sum_{i} F_{A}\left(a_{i}\right) c_{i}$ and we can write $c_{i}=\sum_{j} F_{B}\left(b_{i, j}\right) c_{i, j}$. Thus $c_{i}=\sum_{i, j} F_{A}\left(a_{i}\right) F_{B}\left(b_{i, j}\right) c_{i, j} \in f(A \otimes B) C$. 
Corollary 4.7 The category $\operatorname{Wor}\left(C^{*}\right.$-Alg) is naturally a monoidal category with as monoidal product the $R$-coproduct and with $\mathbb{C}$ as unit.

Proof It is well-known that the category of $C^{*}$-algebras with usual $C^{*}$-morphisms is monoidal with as monoidal product the maximal tensor product. Thus for each three $C^{*}$ algebras $A, B$ and $C$ we have a suitable isomorphism $\psi: A \otimes_{\mu}\left(B \otimes_{\mu} C\right) \rightarrow\left(A \otimes_{\mu}\right.$ $B) \otimes_{\mu} C$. This induces a nondegenerate morphism since $\psi$ is surjective. Hence, we have an isomorphism in $\operatorname{Wor}\left(C^{*}\right.$-Alg).

Theorem 4.8 In the category Wor( $k$-Alg), R-coproducts exist and are given by the nondegenerate tensor product $\widetilde{\otimes}$.

Proof Let $A$ and $B$ be $k$-algebras. We can form the tensor product $A \widetilde{\otimes} B$. We have shown that we have maps

$$
J_{A}: A \longrightarrow \mathcal{M}(A) \longrightarrow \mathcal{M}(A \otimes B)
$$

and

$$
J_{B}: B \longrightarrow \mathcal{M}(B) \longrightarrow \mathcal{M}(A \otimes B)
$$

which are nondegenerate and commuting.

Now let $F_{A}: A \longrightarrow \mathcal{M}(C)$ and $F_{B}: B \longrightarrow \mathcal{M}(C)$ be commuting nondegenerate morphisms. Then we define $H: A \otimes B \longrightarrow \mathcal{M}(C)$ by $H(a \otimes b)=F_{A}(a) F_{B}(b)$. We show that $H(\widetilde{I})=0$, which proves that $h$ descends to a map $F: A \widetilde{\otimes} B \longrightarrow \mathcal{M}(C)$. So take $a \otimes b \in \widetilde{I}$. Then there is some $k \geq 0$ such that for each $y_{1}, \ldots, y_{k} \in A \otimes B$ holds that $y_{1} \ldots y_{k}(a \otimes b)=0$. Now take $c \in C$, by nondegeneracy of $F_{A}$ and $F_{B}$ and by using that $F_{A}$ and $F_{B}$ are commuting, we can write $c$ as

$$
c=\sum_{i_{1}, \ldots, i_{k}} c F_{A}\left(a_{i_{1}}\right) F_{B}\left(b_{i_{1}}\right) \ldots F_{A}\left(a_{i_{k}}\right) F_{B}\left(b_{i_{k}}\right) .
$$

Thus

$$
\begin{aligned}
c F_{A}(a) F_{B}(b) & =c \sum_{i_{1}, \ldots, i_{k}} F_{A}\left(a_{i_{1}} \ldots a_{i_{k}} a\right) F_{B}\left(b_{i_{1}} \ldots b_{i_{k}} b\right) \\
& =c \sum_{i_{1}, \ldots, i_{k}} H\left(\left(a_{i_{1}} \otimes b_{i_{1}}\right) \ldots H\left(a_{i_{k}} \otimes b_{i_{k}}\right)(a \otimes b)\right) \\
& =0 .
\end{aligned}
$$

Since $c$ is arbitrary, it follows that $F_{A}(a) F_{B}(b)=0$, as desired.

The map $F$ is clearly the unique map for which $F_{A}=\bar{F} Q_{A}$ and $F_{B}=\bar{F} Q_{B}$. We must prove that $F$ is nondegenerate. For this, take $c \in C$. Then we can write $c=\sum_{i} F_{A}\left(a_{i}\right) c_{i}$ and we can write $c_{i}=\sum_{j} F_{B}\left(b_{i, j}\right) c_{i, j}$. Thus $c_{i}=\sum_{i, j} F_{A}\left(a_{i}\right) F_{B}\left(b_{i, j}\right) c_{i, j} \in H(A \otimes B) C$.

Corollary 4.9 The category Wor ( $k$-Alg) is naturally a monoidal category with as monoidal product $\widetilde{\otimes}$ and with as unit $k$.

Proof We show that the tensor product is associative as the rest of the statement is obvious. Let $A, B, C$ be nondegenerate $k$-algebras and let $\psi: A \otimes(B \otimes C) \rightarrow(A \otimes B) \otimes C$ be the canonical isomorphism such that $\psi(a \otimes(b \otimes c))=(a \otimes b) \otimes c$. This induces a canonical isomorphism $\widetilde{\psi}: A \widetilde{\otimes}(B \widetilde{\otimes} C) \rightarrow(A \widetilde{\otimes} B) \widetilde{\otimes} C$. Since $\widetilde{\psi}$ is an isomorphism, it is nondegenerate and thus it implements an isomorphism in Wor $(k-\mathbf{A l g})$. 


\subsection{Generalized Colimits}

The following notion generalizes $R$-coproducts and $R$-pushouts:

Definition 4.10 Let $F: I \longrightarrow \mathcal{C}$ be a functor, let $R$ be a co-relation on $\mathcal{C}$ as before, and let $\mathfrak{r}$ be a relation on $\mathrm{Ob}(I)$. An $(\mathfrak{r}, R)$-cocone on $F$ consists of a cocone $\left(C,\left(\varphi_{i}: F(i) \longrightarrow C\right)_{i}\right)$ such that $i \mathfrak{r} j$ implies $\varphi_{i} R \varphi_{j}$. An $(\mathfrak{r}, R)$-colimit on $F$ is a universal cocone on $F$.

Obviously, if it exists, an $(\mathfrak{r}, R)$-colimit of $F$ is unique.

Example 4.11 (1) If $\mathfrak{r}=\varnothing$ and $R$ is the standard co-relation on $\mathcal{C}$, then $(\mathfrak{r}, R)$-colimits are categorical colimits in $\mathcal{C}$.

(2) Let $I=\langle a \quad b\rangle$ be the "coproduct category". If $\mathfrak{r}=\{(a, b)\}$, then an $(\mathfrak{r}, R)$-colimit is an $R$-coproduct. If $\mathfrak{r}=\{(a, b),(b, a)\}$, we call an $(\mathfrak{r}, R)$-colimit a two-sided $R$ coproduct. If $R$ is symmetric, an $R$-coproduct is automatically two-sided.

(3) Let $I=\langle a \leftarrow c \longrightarrow b\rangle$ be the "pushout category". If $\mathfrak{r}=\{(a, b)\}$, then an $(\mathfrak{r}, R)$-colimit is an $R$-pushout. If $\mathfrak{r}=\{(a, b),(b, a)\}$, we call an $(\mathfrak{r}, R)$ colimit a two-sided $R$-pushout. If $R$ is symmetric, an $R$-pushout is automatically two-sided.

(4) Let $I=\langle a\rangle$ be the "object category" with $a \mathfrak{r} a$. The diagram determined by the object $A$ has $(\mathfrak{r}, R)$-colimit $\left(A, 1_{A}: A \longrightarrow A\right)$ if and only if $A$ is commutative with respect to $R$. For $\mathcal{C}=$ Ring the category of unital rings with the commutation co-relation $R$ from Example $4.2(3)$, the $(\mathfrak{r}, R)$-colimit of the diagram determined by the ring $A$ is the canonical morphism $A \longrightarrow A /[A, A]$ where $[A, A]$ is the ideal generated by the commutators $a a^{\prime}-a^{\prime} a$ for $a, a^{\prime} \in A$.

(5) Let $I=\langle b \longrightarrow a\rangle$ be the "morphism category" with $\mathfrak{r}=\{(b, a)\}$ (resp. $\mathfrak{r}=$ $\{(a, b)\})$. The diagram determined by the morphism $f: B \longrightarrow A$ has $(\mathfrak{r}, R)$-colimit $\left(A, 1_{A}: A \longrightarrow A, f: B \longrightarrow A\right)$ if and only if $f$ is central (resp. op-central) with respect to $R$. For $\mathcal{C}=$ Ring the category of unital rings with the commutation co-relation $R$ from Example $4.2(3)$, the $(\mathfrak{r}, R)$-colimit of the diagram determined by the ring map $f: B \longrightarrow A$ is the canonical morphism $A \longrightarrow A /[f(B), A]$ where $[f(B), A]$ is the ideal generated by the commutators $f(b) a-a f(b)$ for $a \in A$ and $b \in B$.

Proposition 4.12 Suppose $\mathcal{C}$ has $R$-coproducts as well as categorical coequalizers. Then $\mathcal{C}$ has R-pushouts.

Proof For morphisms $f: C \longrightarrow A$ and $g: C \longrightarrow B$, consider the $R$-coproduct $\left(Q, s_{A}\right.$ : $A \longrightarrow Q, s_{B}: B \longrightarrow Q$ ) and the categorical equalizer $q: Q \longrightarrow E$ of $s_{A} f$ and $s_{B} g$. By Definition 4.1 (2), $q s_{A}$ and $q s_{B}$ are $R$-related. It is easily seen that they make $E$ into an $R$-pushout of $f$ and $g$.

In order to proceed, it is useful to consider how a co-relation $R$ interacts with certain classes of morphisms. Consider the following weakening of the notion of a pretopology on a category $\mathcal{C}$ : a cover system $\mathcal{T}$ on $\mathcal{C}$ consists of the datum, for every object $C \in \mathcal{C}$, of a collection $\mathcal{T}(C)$ of families of morphisms $\left(f_{i}: C_{i} \longrightarrow C\right)_{i}$. Dually, an co-cover system $\mathcal{S}$ on $\mathcal{C}$ consists of the datum, for every object $C \in \mathcal{C}$, of a collection $\mathcal{S}(C)$ of families of morphisms $\left(g_{i}: C \longrightarrow C_{i}\right)_{i}$. A class of morphisms $\mathbb{T}$ in $\mathcal{C}$ gives rise to a 
cover system $\underline{\mathbb{T}}$ with $\underline{\mathbb{T}}(C)=\{f: D \longrightarrow C \mid f \in \mathbb{T}\}$ and a co-cover system $\overline{\mathbb{T}}$ with $\overline{\mathbb{T}}(C)=\{f: C \longrightarrow D \mid f \in \mathbb{T}\}$.

Definition 4.13 Let $R$ be a co-relation on a category $\mathcal{C}$.

(1) Let $\mathcal{T}$ be a cover system on $\mathcal{C}$. We say that $R$ is $\mathcal{T}$-generated if the following condition holds for morphisms $f: A \longrightarrow C$ and $g: B \longrightarrow C$ in $\mathcal{C}$ and a collection of morphisms $\left(f_{i}: A_{i} \longrightarrow A\right)_{i}$ in $\mathcal{T}(A)$ : if for all $i$ we have $f f_{i} R g$, then we have $f R g$.

If $R$ is a bi-co-relation, we call $R$ bi- $\mathcal{T}$-generated if both $R$ and $R^{\text {op }}$ are $\mathcal{T}$-generated.

(2) Let $\mathcal{S}$ be an co-cover system on $\mathcal{C}$. We say that $R$ is $\mathcal{T}$-cogenerated if the following condition holds for morphisms $f: A \longrightarrow C$ and $g: B \longrightarrow C$ in $\mathcal{C}$ and a collection of morphisms $\left(g_{i}: C \longrightarrow C_{i}\right)_{i}$ in $\mathcal{T}(A)$ : if for all $i$ we have $g_{i} f R g_{i} g$, then we have $f R g$.

Consider the classes Epi of epimorphisms and Mono of monomorphisms in $\mathcal{C}$, the cover system $\mathcal{E}$ pi of jointly epimorphic families and the co-cover system Mono of jointly monomorphic families. Note that the co-relations listed in Example 4.2 are bi$\mathcal{E}$ pi-generated and Mono-op-generated (and hence in particular bi-Epi-generated and

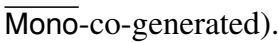

Lemma 4.14 Suppose $\mathcal{C}$ is endowed with a bi-co-relation $R$.

(1) Suppose $R^{\mathrm{op}}$ is Epi-generated. Suppose $f: B \longrightarrow$ A has an $(\mathfrak{r}, R)$-colimit $q: A \longrightarrow$ $A^{\prime}$ as in Example 4.11 (5). Then $q$ is an epimorphism and $q f: B \longrightarrow A^{\prime}$ is central.

(2) Suppose $R$ is bi-Epi-generated. Suppose A has an $(\mathfrak{r}, R)$-colimit $q: A \longrightarrow A^{\prime}$ as in Example 4.11 (4). Then $q$ is an epimorphism and $A^{\prime}$ is commutative.

Under the assumptions of Lemma 4.14, we call $q: A \longrightarrow A^{\prime}$ in (1) the centralizator of $f: B \longrightarrow A$ and $q: A \longrightarrow A^{\prime}$ the commutativizator of $A$.

Proof That $q$ is an epimorphism immediately follows from the universal property of the colimits. In case (1), we consider the epimorphism $q$. By the assumption on $R^{\mathrm{op}}$, from $q f R q$ we deduce $q f R 1_{A^{\prime}}$. In case (2), we twice consider the epimorphism $q: A \longrightarrow A^{\prime}$. By the assumptions upon $R$ and $R^{\text {op }}$, from $q R q$ we deduce $1_{A^{\prime}} R 1_{A^{\prime}}$.

Proposition 4.15 Suppose $\mathcal{C}$ is endowed with a bi-Epi-generated bi-co-relation $R$. Consider morphisms $f: C \longrightarrow A$ and $g: C \longrightarrow B$ with $\overline{t w o}$-sided $R$-coproduct $\left(Q, s_{A}: A \longrightarrow\right.$ $\left.Q, s_{B}: B \longrightarrow Q\right)$ as in Example 4.11 (2).

(1) $s_{A} f=s_{B} g$ is central and op-central.

(2) If $A$ and $B$ are commutative, then so is $Q$.

Proof (1) We show centrality, that is $s_{A} f R 1_{Q}$. Since $\left\{s_{A}, s_{B}\right\}$ is jointly epimorphic, it suffices that $s_{A} f R s_{A}$ and $s_{A} f R s_{B}$. The second relation follows from $s_{A} R s_{B}$, and the first one can be rewritten as $s_{B} g R s_{A}$ which follows from $s_{B} R s_{A}$. 
(2) Using twice the family $\left\{s_{A}, s_{B}\right\}, 1_{Q} R 1_{Q}$ is equivalent to the following four relations: $s_{A} R s_{A}, s_{B} R s_{B}, s_{A} R s_{B}, s_{B} R s_{A}$. The first two follow from commutativity of $A$ and of $B$, the last two follow from the definition of twosided $R$-pushout.

The following example shows that it is not always true that the composition of $R$-pushout diagrams is an $R$-pushout diagram, or that the $R$-pushout of an isomorphism remains an isomorphism.

Example 4.16 Take the category of unital algebras over $\mathbb{R}$ in which $f: A \longrightarrow C$ and $g: B \longrightarrow C$ are $R$-related if and only if they commute. For central morphisms $A \longrightarrow B$ and $A \longrightarrow C$, it is easily checked that the $R$-pushout is given by $B \otimes_{A} C$. Now consider the following diagram, where $\mathbb{H}$ is the quaternion algebra:

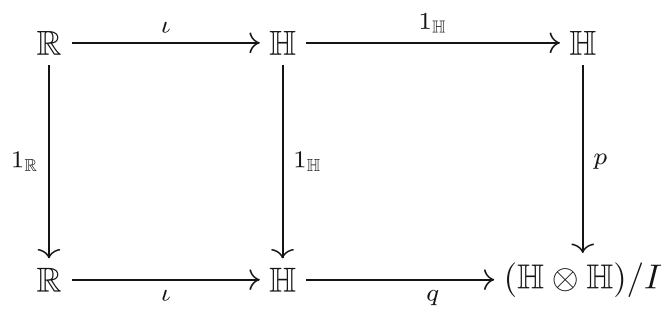

The left diagram is clearly an $R$-pushout diagram. The right diagram is by definition an $R$-pushout diagram, where $I$ is the ideal generated by $h \otimes 1-1 \otimes h$. The composite diagram would only be a pushout diagram if $(\mathbb{H} \otimes \mathbb{H}) / I \cong \mathbb{H}$. This is not the case since otherwise we would have $p=q=1_{\mathbb{H}}$, but the map $1_{\mathbb{H}}: \mathbb{H} \longrightarrow \mathbb{H}$ is not central.

In general, we have the following:

Proposition 4.17 Suppose $R$ is a bi-E $\mathcal{E}$ i-generated bi-co-relation on $\mathcal{C}$. Consider the following composition of diagrams in which $\gamma$ is $R$-central:

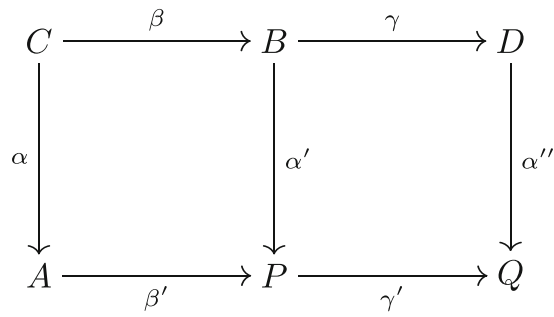

(1) If both squares are two-sided R-pushouts, then so is the composed diagram.

(2) If the left square and the composed diagram are two-sided R-pushouts, then so is the right square.

Proof (1) Obviously, we have $\gamma^{\prime} \beta^{\prime} R \alpha^{\prime \prime}$ since $\gamma^{\prime} R \alpha^{\prime \prime}$. Consider morphisms $f: A \longrightarrow E$ and $g: D \longrightarrow E$ with $f R g$. It follows that $f R g \gamma$, so from the left $R$-pushout we obtain a 
unique morphism $x: P \longrightarrow E$ with $f=x \beta^{\prime}$ and $g \gamma=x \alpha$. If we can show that $x R g$, then we obtain the required unique morphism $x^{\prime}: Q \longrightarrow E$. Since $\left\{\beta^{\prime}, \alpha^{\prime}\right\}$ is jointly epimorphic, it suffices that $x \beta^{\prime} R g$ and $x \alpha R g$. The first relation is $f R g$ which is given. For the second relation, centrality of $\gamma$ gives $\gamma R 1_{D}$, whence $g \gamma R g$ which can be rewritten as $x \alpha R g$ as desired.

(2) If we can show that $\gamma^{\prime} R \alpha^{\prime \prime}$, we can finish the proof in the usual fashion. Along the lines of part (1), we use the jointly epimorphic $\left\{\beta^{\prime}, \alpha^{\prime}\right\}$ to show this.

Example 4.18 In the category of unital $k$-algebras over a commutative ground ring, if in the notations of Proposition $4.17 \alpha, \beta$ and $\gamma$ are central, part (1) of the result corresponds to the familiar change of rings formula $\left(A \otimes_{C} B\right) \otimes_{B} D \cong A \otimes_{C} D$.

Next we give a construction of $R$-pushouts using centralizations and $R$-pushouts of central morphisms.

Proposition 4.19 Suppose $R$ is a bi-E $\mathcal{E}$ pi-generated bi-co-relation on $\mathcal{C}$. Consider morphisms $\alpha: C \longrightarrow A$ and $\beta: C \longrightarrow B$. Suppose the centralizators $q_{A}: A \longrightarrow A^{\prime}$ of $\alpha$ and $q_{B}: B \longrightarrow B^{\prime}$ of $\beta$ exist. Suppose further that $\left(Q, s_{A^{\prime}}: A^{\prime} \longrightarrow Q, s_{B^{\prime}}: B^{\prime} \longrightarrow Q\right)$ is the two-sided R-pushout of $q_{A} \alpha$ and $q_{B} \beta$. Then $\left(Q, s_{A^{\prime}} q_{A}, s_{B^{\prime}} q_{B}\right)$ is the two-sided R-pushout of $\alpha$ and $\beta$.

Proof It suffices to check the universal property. Consider $f: A \longrightarrow D$ and $g: B \longrightarrow D$ with $f R g, g R f$ and $f \alpha=g \beta$. This implies $f \alpha R g$, in other words $g \beta R g$, and $g \beta R f$, in other words $f \alpha R f$. From the universal properties of the centralizators, we thus obtain $f^{\prime}: A^{\prime} \longrightarrow D$ with $f^{\prime} q_{A}=f$ and $g^{\prime}: B^{\prime} \longrightarrow D$ with $g^{\prime} q_{B}=g$. If we can show $f^{\prime} R g^{\prime}$ and $g^{\prime} R f^{\prime}$, we obtain the required morphism $Q \longrightarrow D$. But since both $q_{A}$ and $q_{B}$ are epimorphisms, it suffices that $f^{\prime} q_{A} R g^{\prime} q_{B}$ and $g^{\prime} q_{B} R f^{\prime} q_{A}$ which is part of our assumption of $f$ and $g$.

\subsection{Relations and Generalized Pullbacks}

We can easily dualize the notions of co-relation $R, R$-coproduct and $R$-pushout. Since we will make frequent use of these dual notions, we state them explicitely to fix terminology. For a category $\mathcal{C}$ and an object $C \in \mathcal{C}$, the slice category $C / \mathcal{C}$ is the category of all morphisms with domain $C$.

Definition 4.20 A relation $R$ on a category $\mathcal{C}$ consists of the datum of a relation $R_{C}$ on $\mathrm{Ob}(C / \mathcal{C})$ for every object $C \in \mathcal{C}$, such that the following properties are satisfied:

(1) If $f: C \longrightarrow A$ and $g: C \longrightarrow B$ are $R_{C}$-related and if $h: A \longrightarrow D$ is an arbitrary morphism, then $h f$ and $g$ are $R_{C}$-related. 
(2) If $f: C \longrightarrow A$ and $g: C \longrightarrow B$ are $R_{C}$-related and if $h: B \longrightarrow C$ is an arbitrary morphism, then $f h$ and $g h$ are $R_{B}$-related.

The relation on $\mathcal{C}$ for which every two morphisms with the same codomain are related is called the standard relation.

Definition 4.21 Let $\mathcal{C}$ be a category with a relation $R$ on $\mathcal{C}$.

(1) A morphism $f: A \longrightarrow B$ in $\mathcal{C}$ is $R$-central if $\left(f, 1_{A}\right)$ is in $R$ and $R$-op-central if $\left(1_{A}, f\right)$ is in $R$.

(2) An object $A \in \mathcal{C}$ is $R$-commutative if $\left(1_{A}, 1_{A}\right) \in R$.

The $R$-central morphisms in $\mathcal{C}$ constitute a right ideal in $\mathcal{C}$. The $R$-commutative objects in $\mathcal{C}$ form a full subcategory $\operatorname{Com}(\mathcal{C}) \subseteq \mathcal{C}$. The relation $R$ on $\mathcal{C}$ gives rise to an obvious restricted relation on any subcategory. In particular, the restricted relation on $\operatorname{Com}(\mathcal{C})$ is the standard relation on $\operatorname{Com}(\mathcal{C})$.

Definition 4.22 Let $A$ and $B$ be elements of a category $\mathcal{C}$ with relation $R$. The $R$-product of $A$ and $B$ is an object $P$ together with $R$-related morphisms $p_{A}: P \longrightarrow A$ and $p_{B}$ : $P \longrightarrow B$ such that for every two $R$-related morphisms $q_{A}: Q \longrightarrow A$ and $q_{B}: Q \longrightarrow B$, there is a unique morphism $f: Q \longrightarrow B$ such that $q_{A}=p_{A} f$ and $q_{B}=p_{B} f$.

We denote the $R$-product of $A$ and $B$ by $A \times{ }^{R} B$.

Definition 4.23 Let $f: A \longrightarrow C$ and $g: B \longrightarrow C$ be morphisms in a category $\mathcal{C}$ with relation $R$. The $R$-pullback of $f$ and $g$ is an object $P$ together with $R$-related morphisms $p_{A}: P \longrightarrow A$ and $p_{B}: P \longrightarrow B$ such that $f p_{A}=g p_{B}$ and such that for every two $R$ related morphisms $q_{A}: D \longrightarrow A$ and $q_{B}: D \longrightarrow B$ with $f q_{A}=g q_{B}$ we have that there is a unique morphism $h$ such that $q_{A}=p_{A} h$ and $q_{B}=p_{B} h$.

We denote the $R$-pullback of $f$ and $g$ as above by $A \times{ }_{C}^{R} B$. We call $p_{B}$ the $R$-pullback of $f$ along $g$, and by an $R$-pullback of $f$ we mean an $R$-pullback $p_{B}$ of $f$ along some morphism $g$.

Just like $R$-pushouts, in general $R$-pullbacks do not behave well with respect to compositions and isomorphisms.

The following observation will be useful later on:

Lemma 4.24 Consider a monomorphism $m: Y \longrightarrow Z$ and an $R$-central morphism $g$ : $X \longrightarrow Y$. The following is the $R$-pullback of $m$ along $m g$ :

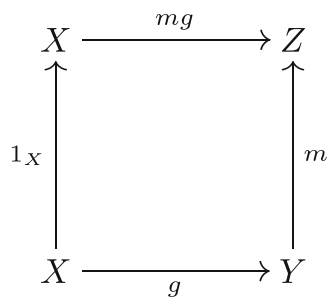


The following terminology may be somewhat confusing, but we prefer to use the words "generated" and "cogenerated" in analogy with the classical meaning of an object $C$ being generated by morphisms landing in $C$ resp. cogenerated by morphisms starting in $C$.

Definition 4.25 Let $R$ be a relation on a category $\mathcal{C}$.

(1) Let $\mathcal{T}$ be a cover system on $\mathcal{C}$. We say that $R$ is $\mathcal{T}$-generated if the following condition holds for morphisms $f: C \longrightarrow A$ and $g: C \longrightarrow B$ in $\mathcal{C}$ and a collection of morphisms $\left(f_{i}: C_{i} \longrightarrow C\right)_{i}$ in $\mathcal{T}(C)$ : if for all $i$ we have $f f_{i} R g f_{i}$, then we have $f R g$.

(2) Let $\mathcal{S}$ be an co-cover system on $\mathcal{C}$. We say that $R$ is $\mathcal{T}$-cogenerated if the following condition holds for morphisms $f: C \longrightarrow A$ and $g: C \longrightarrow B$ in $\mathcal{C}$ and a collection of morphisms $\left(g_{i}: A \longrightarrow A_{i}\right)_{i}$ in $\mathcal{T}(A)$ : if for all $i$ we have $g_{i} f R g$, then we have $f R g$.

If $R$ is a bi-relation, then $R$ is called bi-S-cogenerated if both $R$ and $R^{\text {op }}$ are $\mathcal{S}$-cogenerated.

The following result generalizes well-known stability properties for categorical pullbacks:

Proposition 4.26 Consider the commutative diagram

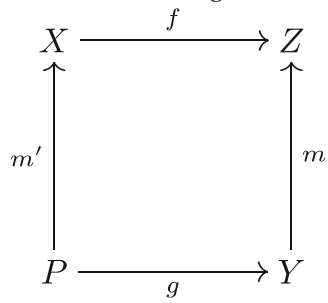

and suppose it is either the R-pullback of $f$ and $m$, or the R-pullback of $m$ and $f$, or the two-sided $R$-pullback of $f$ and $m$.

(1) If $m$ is a monomorphism, then so is $m^{\prime}$.

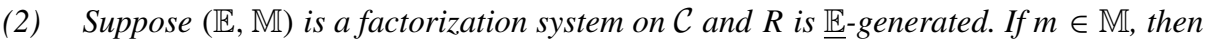
$m^{\prime} \in \mathbb{M}$.

Proof Consider the following commutative diagram

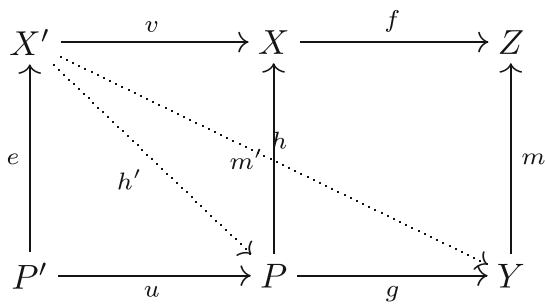

in which the right square is the $R$-pullback of $f$ and $m, m \in \mathbb{M}$ and $e \in \mathbb{E}$. Since $e \perp m$ there is a unique morphism $h: X^{\prime} \longrightarrow Y$ with $m h=f v$ and $h e=g u$. In order to apply 
the $R$-pullback property, we need $v R h$. We have $h e=g u$ and $v e=m^{\prime} u$, from $m^{\prime} R g$ we deduce $m^{\prime} u R g u$ in other words veRhe. Since $R$ is $\underline{\mathbb{E}}$-generated, we have $v R h$ so we get a unique map $h^{\prime}: X^{\prime} \longrightarrow P$ with $m^{\prime} h^{\prime}=v$ and $g h^{\prime}=h$. Now $m^{\prime} h^{\prime} e=v e=m^{\prime} u$ is equivalent to $h^{\prime} e=u$ since $\mathbb{M} \subseteq$ Mono. This finishes the proof of the first statement. The proofs of the other statements are similar.

\section{Tensor Functional Topology}

The basic idea of functional topology is that a certain amount of "topology" can be developed in a category based upon axiomatically defined classes of morphisms. After this idea originated in the seventies in work of Herrlich, Manes and Penon [10], [18], [21] [20], since the nineties the subject received a lot of attention in work of Herrlich, Salicrup, Strecker, Clementino, Giuli, Tholen, Hofmann and others with applications to Birkhoff closure spaces, uniform spaces, topological groups, locales, approach spaces, lax algebras and schemes [12], [4], [22] [5], [6], [13], [16]. In this section, we adapt some of these approaches to a monoidal context, or, more generally, to the context of a category endowed with a relation $R$ in the sense of Definition 4.20. We mainly focuss our attention on the following two approaches:

- a minimal approach inspired by [13], taking an $R$-proper class of so-called proper morphisms as primary (Section 5.1). This allows for the definition of compact objects (Section 5.4), of commutative Hausdorff objects (Section 5.4) and of separated morphisms with commutative domain (Section 5.2). Many familiar relations between these notions hold true, sometimes under suitable centrality conditions. This approach can be refined by adding a second class $\mathbb{F}_{0}$ of closed immersions to the data (Section 5.3).

- $\quad$ an approach building on [5], [16], in the presence of a factorization system (E, $\mathbb{M})$. This involves the definition of a closed class $\mathbb{F}$ of closed morphisms based upon an auxiliary $R$-proper class $\mathbb{F}_{0}$ of closed immersions and an auxiliary closed class $\mathbb{P}$ of surjections, together constituting a co-called $(\mathbb{E}, \mathbb{M})$-closed structure $\left(\mathbb{P}, \mathbb{F}_{0}\right)$. If $\mathbb{F}$ is moreover an $R$-proper class, we call $\left(\mathbb{P}, \mathbb{F}_{0}\right)$ an $(\mathbb{E}, \mathbb{M})$-proper structure with proper morphisms $\mathbb{F}$ (Section 5.5).

If $R$ is such that $R$-pullback diagrams can naturally be composed, and $R$-pullbacks of identity morphims remain identity morphisms, then the results from [16] integrally go through. In this section however, we concentrate on the results which still hold true more generally. Unlike in the classical case, there is no standard way of associating an $R$-proper class to a closed class, so the most natural thing to do in this setup is start at once with the class of morphisms one would like to view as "proper", and show by hand that it is an $R$ proper class. Even in the second approach, there is no general way of checking whether the closed class $\mathbb{F}$ we obtain from $(\mathbb{E}, \mathbb{M})$ and $\left(\mathbb{P}, \mathbb{F}_{0}\right)$ is actually $R$-proper (that is we have an $(\mathbb{E}, \mathbb{M})$-proper structure). On the other hand, if we start from a closed class $\mathbb{F}$ and we have a factorization system $(\mathbb{E}, \mathbb{M})$ with $\mathbb{M} \subseteq \mathbb{F}$, then under the additional conditions that $R$ is

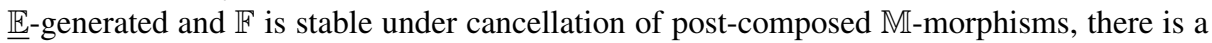
canonical way of associating the closed structure $(\mathbb{E} \cap \mathbb{F}, \mathbb{M}$ ) to it (see Propositions 5.18 and 5.19), which is at the other extreme from the case $\mathbb{P}=\mathbb{E}$ corresponding to the setup in [5].

From now on, we will always work in a category $\mathcal{C}$ with a fixed relation $\boldsymbol{R}$. 


\subsection{Proper Morphisms}

Let $\mathcal{C}$ be a category with $R$-pullbacks. We make use of the standard classes Mor of all morphisms, Iso of isomorphisms, Mono of monomorphisms and Epi of epimorphisms.

In general, one is interested in classes $\mathbb{F}$ of morphisms satisfying some of the following stability properties:

(1) Iso $\subseteq \mathbb{F}$;

(2) $\mathbb{F}$ is closed under composition;

(3) $\quad \mathbb{F}$ is $R$-pullback stable, that is every $R$-pullback of $f \in \mathbb{F}$ along an arbitrary morphism is again in $\mathbb{F}$.

If $\mathbb{F}$ satisfies (1) and (2), we call $\mathbb{F}$ a closed class. If $\mathbb{F}$ satisfies (1), (2) and (3), it is called an $R$-proper class.

Definition 5.1 Let $\mathbb{F}$ be a class of morphisms. We say that a morphism $g$ is $\mathbb{F}$-proper if every $R$-pullback of $g$ exists and is in $\mathbb{F}$.

We thus obtain the class $\mathbb{F}$ - Prop of $\mathbb{F}$-proper morphisms.

Lemma 5.2 Let $\mathbb{F}$ be a class of morphisms.

(1) If $\mathbb{F} \subseteq \mathbb{G}$ then $\mathbb{F}-$ Prop $\subseteq \mathbb{G}-$ Prop.

(2) If $\mathbb{F}_{0} \subseteq \mathbb{F}$ with $\mathbb{F}_{0} R$-pullback-stable, then each morphism in $\mathbb{F}_{0}$ is $\mathbb{F}$-proper.

Unlike in the familiar situation for the standard relation, in general $\mathbb{F}$ - Prop fails to be a proper class. We have:

Lemma 5.3 Suppose $R$ is symmetric and Mono-cogenerated. Let $\mathbb{F}$ be a class of morphisms. Consider morphisms $f: X \longrightarrow Y$ and $g: Y \longrightarrow Z$. If $f, g \in \mathbb{F}-$ Prop and $f$ is central, then $g f \in \mathbb{F}-\operatorname{Prop}$ (and $g f$ is central).

Due to the failure of $\mathbb{F}$ - Prop to be proper in general, it is more natural to start at once from a proper class $\mathbb{F}$ considered as "proper morphisms", as is done in [13]. Obviously, a proper class $\mathbb{F}$ satisfies $\mathbb{F}=\mathbb{F}$ - Prop.

\subsection{Separated Morphisms}

Let $\mathcal{C}$ be a category with $R$-pullbacks. For a morphism $g: X \longrightarrow Y$, we consider the following $R$-pullback diagram:

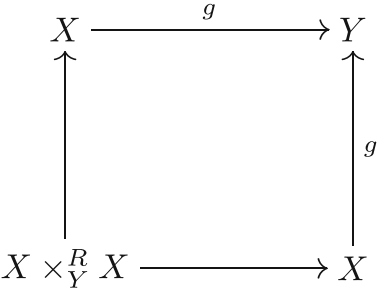

If $X$ is $R$-commutative, we obtain a unique diagonal morphism

$$
\Delta_{g}=\left(1_{X}, 1_{X}\right): X \longrightarrow X \times{ }_{Y}^{R} X
$$


from the universal property of an $R$-pullback.

We can now introduce separated morphisms:

Definition 5.4 Let $\mathbb{F}$ be a class of morphisms. Let $g: X \longrightarrow Y$ be a morphism such that $X$ is a commutative object.

(1) We say that $g$ is $\mathbb{F}$-separated if the diagonal $\Delta_{g} \in \mathbb{F}$.

(2) We say that $g$ is $\mathbb{F}$-perfect if it is $\mathbb{F}$-proper and $\mathbb{F}$-separated.

We thus obtain the corresponding classes $\mathbb{F}$ - Sep of $\mathbb{F}$-separated morphisms and $\mathbb{F}$-Perf of $\mathbb{F}$-perfect morphisms. A class $\mathbb{F}$ is called separating if every $\mathbb{F}$-separated morphism $g$ satisfies $\Delta_{g} \in \mathbb{F}$ - Prop. Obviously, every proper class is separating.

Lemma 5.5 Let $\mathbb{F}$ be a class of morphisms.

1. If $\mathbb{F} \subseteq \mathbb{G}$ then $\mathbb{F}-\operatorname{Sep} \subseteq \mathbb{G}-$ Sep.

2. Suppose Iso $\subseteq \mathbb{F}$. Every monomorphism $m: X \longrightarrow Y$ with $X$ commutative is $\mathbb{F}$ separated.

Proof (2) For a monomorphism $m: X \longrightarrow Y$ with $X$ commutative, the diagram

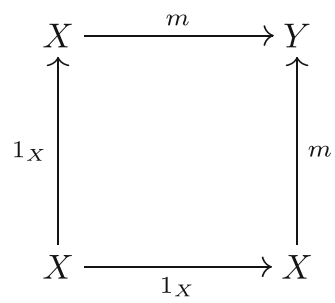

is an $R$-pullback by Lemma 4.24, whence $\Delta_{m}=1_{X}: X \longrightarrow X$ is in $\mathbb{F}$.

The following generalizes [16, Lemma 2.6]:

Lemma 5.6 Suppose $R$ is symmetric and Mono-cogenerated. Let $\mathbb{F}$ be a proper class of morphisms. Consider morphisms $f: X \longrightarrow Y$ and $g: Y \longrightarrow Z$. If $f$ is central, $g \in \mathbb{F}-\operatorname{Sep}$ and $g f \in \mathbb{F}$, then $f \in \mathbb{F}$.

Proof Since $f$ and $\Delta_{g}$ are central, the proof from [16, Lemma 2.6] goes through.

\subsection{Closed and Proper Pairs}

Definition 5.7 Consider a proper class of monomorphisms $\mathbb{F}_{0} \subseteq$ Mono and a closed class $\mathbb{F}$ with $\mathbb{F}_{0} \subseteq \mathbb{F}$. The pair $\left(\mathbb{F}_{0}, \mathbb{F}\right)$ is called a closed pair, the morphisms in $\mathbb{F}$ are called closed morphisms and the morphisms in $\mathbb{F}_{0}$ are called closed immersions. Is $\mathbb{F}$ is moreover a proper class, then $\left(\mathbb{F}_{0}, \mathbb{F}\right)$ is called a proper pair and the morphisms in $\mathbb{F}$ are called proper morphisms.

A closed pair is called separating if for every $\mathbb{F}$-separated morphism $g: X \longrightarrow Y$, we have $\Delta_{g} \in \mathbb{F}_{0}$. 
Proposition 5.8 Assume that $\mathcal{C}$ is a category with $R$-pullbacks. If $\mathbb{H}$ is a proper class, then $(\mathbb{H} \cap \mathrm{Mono}, \mathbb{H})$ is a separating proper pair.

Proof By Proposition 4.26 (1), Mono is a proper class and $\mathbb{H} \cap$ Mono is a proper class of monomorphisms. The resulting proper pair is separating since the diagonal of any morphism is a monomorphism.

\subsection{Compact and Hausdorff Objects}

Suppose $\mathcal{C}$ has a final object 1 . The unique morphism from an object $X$ to 1 is denoted by $!_{X}: X \longrightarrow 1$.

We will define compact and separated objects at once with respect to a proper class, thus avoiding some of the problems that arise if one starts from a closed class.

Definition 5.9 Let $\mathbb{H}$ be a proper class, morphisms of which are called proper morphisms.

(1) An object $X \in \mathcal{C}$ is $\mathbb{H}$-compact if the morphism $!_{X}: X \longrightarrow 1$ is proper.

(2) A commutative object $X \in \mathcal{C}$ is $\mathbb{H}$-Hausdorff if $!_{X}: X \longrightarrow 1$ is $\mathbb{H}$-separated.

Proposition 5.10 Suppose $\mathbb{F}$ is a closed class for which $\mathbb{H}=\mathbb{F}$ - Prop is a proper class.

(1) An object $X \in \mathcal{C}$ is $\mathbb{H}$-compact if and only if for every $Y \in \mathcal{C}$ we have that the second projection $p_{2}: X \times{ }^{R} Y \longrightarrow Y$ is in $\mathbb{F}$.

(2) If $\mathbb{F}$ is separating, then a commutative object $X \in \mathcal{C}$ is $\mathbb{H}$-Hausdorff if and only if $!_{X}: X \longrightarrow 1$ is $\mathbb{F}$-separated.

In the category Top with $\mathbb{F}$ the usual closed maps and $\mathbb{H}=\mathbb{F}$ - Prop the usual proper maps, the $\mathbb{H}$-compact and $\mathbb{H}$-Hausdorff objects are the usual compact and Hausdorff spaces.

We now give some stability properties of compact objects.

Proposition 5.11 Let $\mathbb{H}$ be a proper class.

(1) If $f: X \longrightarrow Y$ is proper and $Y$ is $\mathbb{H}$-compact, then $X$ is $\mathbb{H}$-compact too.

(2) If $X$ and $Y$ are $\mathbb{H}$-compact, then so is $X \times{ }^{R} Y$.

Proof (1) is clear. (2) For $X$ and $Y$ are compact, consider $p_{1}: X \times{ }^{R} Y \longrightarrow X$ and $p_{2}$ : $X \times^{R} Y \longrightarrow Y$. Since $!_{X}: X \longrightarrow 1$ is in $\mathbb{H}$, so is its pullback $p_{2}$. Now also $!_{Y}: Y \longrightarrow 1$ is in $\mathbb{H}$, hence so is the composition $!_{X \times{ }^{R} Y}=!_{Y} p_{2}: X \times{ }^{R} Y \longrightarrow 1$.

The following corollary expresses the well-known topological statement that a closed subspace of a compact space is again compact:

Proposition 5.12 Suppose $\left(\mathbb{F}_{0}, \mathbb{F}\right)$ is a closed pair for which $\mathbb{H}=\mathbb{F}$ - Prop is a proper class. If $f: X \longrightarrow Y$ is in $\mathbb{F}_{0}$ and $Y$ is $\mathbb{H}$-compact, then $X$ is $\mathbb{H}$-compact too. 
Finally, we can recover the famous topological statement that a continuous morphism from a compact to a Hausdorff space is proper.

Proposition 5.13 Let $\mathbb{H}$ be a proper class. Consider a central morphism $f: X \longrightarrow Y$ with $Y$ commutative. If $X$ is $\mathbb{H}$-compact and $Y$ is $\mathbb{H}$-Hausdorff, then $f$ is proper.

Proof This follows from Lemma 5.6 by taking $g=!_{Y}$.

\section{$5.5(\mathbb{E}, \mathbb{M})$-Closed Structures}

Let $\mathcal{C}$ be a category with $R$-pullbacks endowed with a factorization system $(\mathbb{E}, \mathbb{M})$ with $\mathbb{M} \subseteq$ Mono. From now on, we denote the $(\mathbb{E}, \mathbb{M})$-factorization of a morphism $f: X \longrightarrow Y$ by

$$
X \underset{\varepsilon(f)}{\longrightarrow} f(X) \underset{\mu(f)}{\longrightarrow} Y
$$

Definition 5.14 An $(\mathbb{E}, \mathbb{M})$ )-closed structure $\left(\mathbb{P}, \mathbb{F}_{0}\right)$ (or simply closed structure if $(\mathbb{E}, \mathbb{M})$ is understood) consists of:

(a) An $R$-proper class $\mathbb{F}_{0} \subseteq \mathbb{M}$ of closed immersions.

(b) A closed class $\mathbb{P}$ of surjections.

With respect to a closed structure, a morphism $f: X \longrightarrow Y$ is called closed if and only if for every $m: X^{\prime} \longrightarrow X$ in $\mathbb{F}_{0}$, we have $\varepsilon(f m) \in \mathbb{P}$ and $\mu(f m) \in \mathbb{F}_{0}$. The class of closed morphisms is denoted by $\mathbb{F}$. The closed structure is called separating if the following condition is satisfied.

(c) For any morphism $f: A \longrightarrow B$ with $A$ commutative, it holds that if the diagonal $\Delta_{f}$ of $f$ is in $\mathbb{F}$, then it is in $\mathbb{F}_{0}$.

The closed structure is called a proper structure if the class $\mathbb{F}$ is $R$-proper, and in this case the closed morphisms are also called proper morphisms.

The following is an adaptation of [16, Proposition 3.13]:

Proposition 5.15 Let $\left(\mathbb{P}, \mathbb{F}_{0}\right)$ be an $(\mathbb{E}, \mathbb{M})$-closed structure. We have:

(1) $\mathbb{F}$ is closed under compositions, thus $\left(\mathbb{F}_{0}, \mathbb{F}\right)$ is a closed pair.

(2) $\quad \mathbb{F}_{0}=\mathbb{F} \cap \mathbb{M}$.

(3) If we have $m g \in \mathbb{F}$ for a monomorphism $m$ and an $R$-central morphism $g$, then also $g \in \mathbb{F}$.

If $\left(\mathbb{P}, \mathbb{F}_{0}\right)$ is a separating $(\mathbb{E}, \mathbb{M})$-closed structure, then $\left(\mathbb{F}, \mathbb{F}_{0}\right)$ is a separating closed pair.

Remarks 5.16 (1) Suppose we take $\mathbb{P}=\mathbb{E}$. Then a morphism $f: X \longrightarrow Y$ is closed if and only if for $m: X^{\prime} \longrightarrow X$ in $\mathbb{F}_{0}$ we have $\mu(f m) \in \mathbb{F}_{0}$. Thus, this is precisely the situation of [5].

(2) Suppose we take $\mathbb{F}_{0}=\mathbb{M}$. Then a morphisms $f: X \longrightarrow Y$ is closed if and only if for $m: X^{\prime} \longrightarrow X$ in $\mathbb{F}_{0}$ we have $\varepsilon(f m) \in \mathbb{P}$. 
The following is an adaptation of [16, Proposition 3.15]:

Proposition 5.17 Let $\left(\mathbb{E}, \mathbb{F}_{0}\right)$ be an $(\mathbb{E}, \mathbb{M})$-closed structure. Suppose in an R-pullback

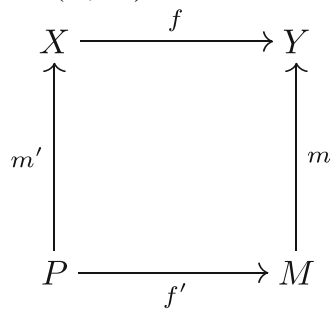

of $m \in \mathbb{F}_{0}$ and $f \in \mathbb{E}$, we have $f^{\prime} \in \mathbb{E}$. If $g f \in \mathbb{F}$ and $f \in \mathbb{E}$, then $g \in \mathbb{F}$.

Proof Take $f: X \longrightarrow Y$ and $g: Y \longrightarrow Z$ morphisms. Let $g f \in \mathbb{F}$ with $f \in \mathbb{E}$. Let $m: M \longrightarrow Y$ be an arbitrary morphism in $\mathbb{F}_{0}$. We need to show that $\mu(\mathrm{gm}) \in \mathbb{F}_{0}$. For this, we take the $R$-pullback of $m$ and $f$. We have the following diagram:

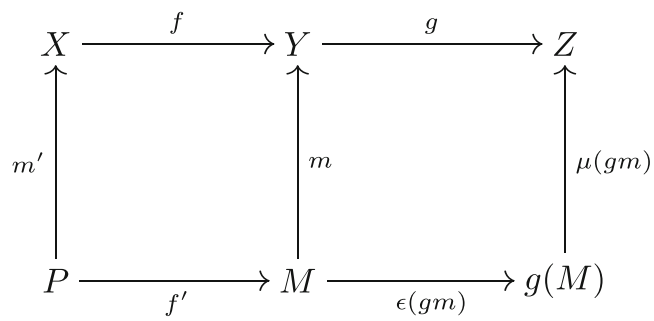

Since $\mathbb{F}_{0}$ is stable under $R$-pullbacks, we get that $m^{\prime} \in \mathbb{F}_{0}$. Since $f \in \mathbb{E}$, the assumptions of the proposition ensure that $f^{\prime} \in \mathbb{E}$. Thus $\mu\left(g f m^{\prime}\right)=\mu(\mathrm{gm})$. But since $g f$ is closed, this is an element of $\mathbb{F}_{0}$.

In some situations we have $\mathbb{M}=\mathbb{F}_{0}$. The following two propositions deal with this case:

Proposition 5.18 Let $(\mathbb{P}, \mathbb{M})$ be an $(\mathbb{E}, \mathbb{M})$-closed structure and let $\mathbb{F}$ be the class of closed morphisms with respect to this closed structure.

(1) If we have $m g \in \mathbb{F}$ for $m \in \mathbb{M}$, then also $g \in \mathbb{F}$.

(2) $\quad \mathbb{F}$ is closed under $R$-pullbacks along morphisms in $\mathbb{M}$.

Proof (1) Consider $m: Y \longrightarrow Z$ in $\mathbb{M}$ and $f: X \longrightarrow Y$ with $m g \in \mathbb{F}$. Consider $m^{\prime}: X^{\prime} \longrightarrow X$ in $\mathbb{M}$. We have $\mu\left(m g m^{\prime}\right)=m \mu\left(g m^{\prime}\right)$ and $\epsilon\left(m g m^{\prime}\right)=\epsilon\left(g m^{\prime}\right)$. Since $m g \in \mathbb{F}$, the latter is in $\mathbb{P}$.

(2) Consider the pullback square 


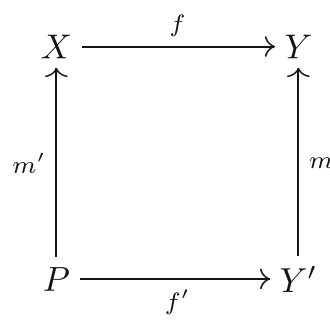

with $f \in \mathbb{F}, m \in \mathbb{M}$. Then $m f^{\prime}=f m^{\prime} \in \mathbb{F}$, whence by (1), $f^{\prime} \in \mathbb{F}$.

Conversely, we have:

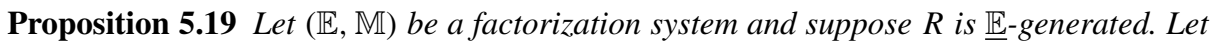
$\mathbb{F}$ be a closed class with $\mathbb{M} \subseteq \mathbb{F}$ and assume that $m g \in \mathbb{F}$ for $m \in \mathbb{M}$ implies $g \in \mathbb{F}$. Then $(\mathbb{E} \cap \mathbb{F}, \mathbb{M})$ is an $(\mathbb{E}, \mathbb{M})$-closed pair, and $\mathbb{F}$ is its class of closed morphisms.

Proof Obviously $\mathbb{E} \cap \mathbb{F}$ is a closed class, and by Proposition 4.26, $\mathbb{M}$ is $R$-proper. It remains to show that $\mathbb{F}$ is the associated class of closed morphisms. Assume that $f \in \mathbb{F}$ and $m \in \mathbb{M}$, then it obviously holds that $\mu(f m) \in \mathbb{M}$ and $\epsilon(f m) \in \mathbb{E}$. But since $\mu(f m) \epsilon(f m)=f m \in$ $\mathbb{F}$, it follows by the hypothesis that $\epsilon(f m) \in \mathbb{F}$.

Conversely, assume that $f: A \longrightarrow B$ is closed with respect to the $(\mathbb{E}, \mathbb{M})$-closed structure $(\mathbb{E} \cap \mathbb{F}, \mathbb{M})$. Since $1_{A} \in \mathbb{M}$, it follows that $f=f 1_{A}=\epsilon\left(f 1_{A}\right) \in \mathbb{F}$. We also have $\mu\left(f 1_{A}\right) \in \mathbb{M} \subseteq \mathbb{F}$. Thus it follows that $f \in \mathbb{F}$ by composition.

\section{Tensor Functional Topology in Woronowicz Categories}

In this section, we show that the opposite Woronowicz categories of both associative algebras (Section 6.2) and $C^{*}$-algebras (Section 6.3) can naturally be endowed with proper structures. In each case, this entails three main steps:

(1) The definition of an $R$-proper class $\mathbb{F}$ of proper morphisms;

(2) The definition of a factorization system $(\mathbb{E}, \mathbb{M})$;

(3) The verification that the conditions of Proposition 5.19 are fulfilled and thus $(\mathbb{E} \cap$ $\mathbb{F}, \mathbb{M})$ is an $(\mathbb{E}, \mathbb{M})$-closed structure.

In each case, compact objects correspond precisely to unital algebras, and all commutative algebras are Hausdorff.

In the $C^{*}$-algebra case, restricting the opposite Woronowicz category to the commutative objects yields a category equivalent to locally compact Hausdorff spaces with continuous maps. We show that the restriction of $(\mathbb{E}, \mathbb{M})$ to this category coincides precisely with the factorization system of dense maps and closed embeddings. Further, the class $\mathbb{F}$ restricts precisely to the proper continuous maps.

\subsection{Topological Situations}

In the category Top of topological spaces and continuous maps, let $\mathbb{F}$ be the class of the usual closed maps. The standard factorization system $\left(\mathbb{E}^{0}, \mathbb{M}^{0}\right)$ has $\mathbb{E}^{0}$ given by the surjections and $\mathbb{M}^{0}$ given by the embeddings. It is well known that taking $\mathbb{F}_{0}$ to be the closed 
embeddings and putting $\mathbb{P}^{0}=\mathbb{E}$ yields a separating closed structure describing the closed maps $\mathbb{F}[5]$.

In [16], we alternatively consider the factorization system $(\mathbb{E}, \mathbb{M})$ with $\mathbb{E}$ given by the dense maps and $\mathbb{M}$ given by the closed immersions. Put $\mathbb{F}_{0}=\mathbb{M}$. Clearly, we are in the situation of Proposition 5.19 and $\mathbb{P}=\mathbb{E} \cap \mathbb{F}$ corresponds to the usual class of surjections. We thus obtain a separating closed structure $(\mathbb{P}, \mathbb{M})$ describing the closed maps $\mathbb{F}$.

Let CHd $\subseteq$ Top be the full subcategory of compact Hausdorff spaces. The factorization systems $\left(\mathbb{E}^{0}, \mathbb{M}^{0}\right)$ and $(\mathbb{E}, \mathbb{M})$ both restrict to $\mathbf{C H d}$ and coincide on this category, corresponding to the fact that every morphism is closed.

Let LcHd $\subseteq$ Top be the full subcategory of locally compact Hausdorff spaces. In this case only the factorization system $(\mathbb{E}, \mathbb{M})$ restricts to LcHd, and taking $\mathbb{F}_{0}=\mathbb{M}$ and $\mathbb{P}$ the surjections again yields a separating closed structure describing the closed maps in LcHd.

\subsection{Associative Algebras}

Let $k$ be a commutative ring. In this section we develop functional topology on the opposite Woronowicz category Wor $(k \text {-Alg })^{\text {op }}$ from Section 3. For a morphism $f: A \longrightarrow B$ in $\operatorname{Wor}(k-\mathrm{Alg})^{\text {op }}$, we write $F: B \longrightarrow \mathcal{M}(A)$ for the associated Woronowicz-morphism. We make the following definitions for $f: A \longrightarrow B$ :

- $\quad f$ is in $\mathbb{F}$ if $F(B) \subseteq A$;

- $f$ is in $\mathbb{M}$ if $F(B)=A$;

- $f$ is in $\mathbb{E}$ if $F$ is injective.

Lemma 6.1 $\mathbb{F}$ is an R-proper class.

Proof It suffices to check that $\mathbb{F}$ is stable under $R$-pullbacks. Consider $F: B \longrightarrow \mathcal{M}(A)$ and $G: B \longrightarrow \mathcal{M}(C)$ arbitrary such that $F(B) \subseteq A$. Let us introduce some notation:

- We let $q: A \otimes C \longrightarrow A \widetilde{\otimes} C$ be the canonical quotient map.

- We let $\widetilde{I}$ be the ideal of $A \widetilde{\otimes} C$ generated by $F(b) a \otimes c-a \otimes G(b) c$ and $a F(b) \otimes c-a \otimes$ $c G(b)$, for arbitrary elements $a \in A, b \in B$ and $c \in C$. We let $p: A \widetilde{\otimes} C \longrightarrow(A \widetilde{\otimes} C) / \widehat{I}$ be the quotient map.

- We let $F^{\prime}: C \longrightarrow \mathcal{M}(C) \longrightarrow \mathcal{M}(A \widetilde{\otimes} C / \widehat{I})$ be defined as $F^{\prime}(c)=\left(\lambda^{\prime}, \rho^{\prime}\right)$, where $\lambda^{\prime}\left(p\left(q\left(a^{\prime} \otimes c^{\prime}\right)\right)\right)=p\left(q\left(a^{\prime} \otimes c c^{\prime}\right)\right)$ and $\rho^{\prime}\left(p\left(q\left(a^{\prime} \otimes c^{\prime}\right)\right)\right)=p\left(q\left(a^{\prime} \otimes c^{\prime} c\right)\right)$.

- We let $G^{\prime}: A \longrightarrow \mathcal{M}(C) \longrightarrow \mathcal{M}(A \widetilde{\otimes} C / \widehat{I})$ be defined $G^{\prime}(a)=\left(\lambda^{\prime}, \rho^{\prime}\right)$, where $\lambda^{\prime}\left(p\left(q\left(a^{\prime} \otimes c^{\prime}\right)\right)\right)=p\left(q\left(a a^{\prime} \otimes c^{\prime}\right)\right)$ and $\rho^{\prime}\left(p\left(q\left(a^{\prime} \otimes c^{\prime}\right)\right)\right)=p\left(q\left(a^{\prime} a \otimes c^{\prime}\right)\right)$.

The following is then the $R$-pullback diagram:

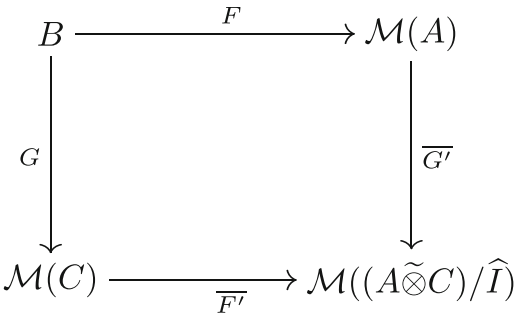


We need to prove that $\overline{F^{\prime}}(c)$ is in $(C \otimes A) / J$. But since $G$ is nondegenerate, we have that $G(B) C=C$. Hence we can write $c=\sum_{n} G\left(b_{n}\right) c_{n}$. Thus

$$
\overline{F^{\prime}}(c)=\sum_{n} \overline{F^{\prime}}\left(G\left(b_{n}\right) c_{n}\right) .
$$

Note that $F\left(b_{n}\right) \in A$ by hypothesis on $F$. Thus by definition of $\overline{F^{\prime}}$, we have

$$
\overline{F^{\prime}}\left(G\left(b_{n}\right) c_{n}\right)=\left(\lambda^{\prime}, \rho^{\prime}\right)
$$

where

$$
\begin{aligned}
\lambda^{\prime}\left(p\left(q\left(b^{\prime} \otimes c^{\prime}\right)\right)\right) & =p\left(q\left(b^{\prime} \otimes G\left(b_{n}\right) c_{n} c^{\prime}\right)\right) \\
& =p\left(q\left(F\left(b_{n}\right) b^{\prime} \otimes c_{n} c^{\prime}\right)\right) \\
& =\lambda_{F\left(b_{n}\right) \otimes c_{n}}\left(p\left(q\left(b^{\prime} \otimes c^{\prime}\right)\right)\right) .
\end{aligned}
$$

Hence $\lambda^{\prime}=\lambda_{F\left(b_{n}\right) \otimes c_{n}}$, and similarly $\rho^{\prime}=\rho_{F\left(b_{n}\right) \otimes c_{n}}$. This is thus in $(B \widetilde{\otimes} C) / \widehat{I}$.

Lemma 6.2 The classes $(\mathbb{E}, \mathbb{M})$ form a factorization system.

Proof (1) $\mathbb{E}$ and $\mathbb{M}$ are closed under composition with isomorphisms.

This is obvious.

(2) Every morphism $f$ decomposes as $f=m e$, with $m \in \mathbb{M}$ and e $\in \mathbb{E}$.

In Wor $(k$-Alg), for every morphism $f: A \longrightarrow \mathcal{M}(B)$, we can decompose it as $A \stackrel{e}{\rightarrow} A / \operatorname{Ker}(f) \stackrel{m}{\rightarrow} \mathcal{M}(B)$. This induces a suitable factorization on the dual category.

(3) Every e $\in \mathbb{E}$ is orthogonal to every $m \in \mathbb{M}$.

Consider a commutative diagram in $\mathbf{W o r}(k-\mathbf{A l g})$ :

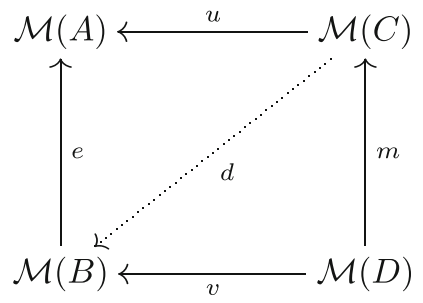

Since $e(D)=C$, we find for each $x \in C$ that there exists an $y \in D$ such that $m(y)=x$. Then we define $d(x)=v(y)$. This is a well-defined morphism since $e$ is injective. It is obvious that $d$ is nondegenerate since $v$ is nondegenerate.

Proposition $6.3(\mathbb{E} \cap \mathbb{F}, \mathbb{M})$ is a separating $(\mathbb{E}, \mathbb{M})$-proper structure on Wor $(k \text {-Alg })^{\text {op }}$ with $\mathbb{F}$ as proper maps. With respect to the proper class $\mathbb{F}$ :

(1) An object A is compact if and only if it is a unital k-algebra.

(2) An object $A$ is Hausdorff if and only if it is a commutative k-algebra.

(3) All morphisms with commutative domain are separated.

Proof By Lemma 6.2, (E, $\mathbb{M}$ ) is a factorization system and by Lemma 6.1, $\mathbb{F}$ is a proper class. We verify that we can apply Proposition 5.19. For this, we must first verify that $R$ is 


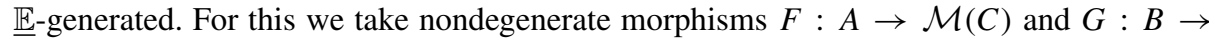
$\overline{\mathcal{M}}(C)$, and we take a nondegenerate injection $F^{\prime}: C \rightarrow \mathcal{M}(D)$. Assume that $\overline{F^{\prime}} F R \overline{F^{\prime}} G$ for each $i$, this means that for each $a \in A$ and $b \in B$, we have $\bar{F}^{\prime}(F(a))$ commutes with $\bar{F}^{\prime}(G(b))$. Since $\overline{F^{\prime}}$ is an injection, this implies directly that $F(a)$ commutes with $G(b)$ and thus that $F R G$.

Next, we must verify the other condition of Proposition 5.19 that $m g \in \mathbb{F}$ and $m \in \mathbb{M}$ implies that $g \in \mathbb{F}$. Dually, this comes down to taking nondegenerate morphisms $M: A \rightarrow$ $\mathcal{M}(B)$ and $G: B \rightarrow \mathcal{M}(C)$ such that $\bar{G} M(A) \subseteq C$ and $M(A)=B$. To verify that $G(B) \subseteq C$, take some $b \in B$. Then there is some $a \in A$ such that $M(a)=b$. Then $G(b)=\bar{G}(b)=\bar{G}(M(a)) \in C$. This verifies the conditions of Proposition 5.19.

(1) The initial object of the category Wor $(k-\mathbf{A l g})^{\mathrm{op}}$ is $k$. A Woronowicz-morphism from $k$ to $A$ is then a nondegenerate morphism $F: k \longrightarrow \mathcal{M}(A)$. Demanding that this morphism is proper is exactly asking that $F(k) \subseteq A$. This is true if and only if $A$ contains a unit.

(2) Let $A$ be a commutative object, that is a commutative $k$-algebra. The diagonal morphism is represented by $D: A \widetilde{\otimes} A \longrightarrow \mathcal{M}(A)$ such that $D(a \otimes b)=a b$. Since the algebra is nondegenerate, we obtain that $D(A \otimes A)=A$.

(3) Let $A$ be a commutative object, that is a commutative $k$-algebra and let $G: B \rightarrow$ $\mathcal{M}(A)$ be the dual of a morphism in $\operatorname{Wor}(k-\mathbf{A l g})^{\text {op }}$. The diagonal is given by $D$ : $A \widetilde{\otimes}_{B} A \rightarrow \mathcal{M}(A)$ such that $D(a \otimes b)=a b \in A$. This diagonal thus obviously satisfies the condition $D\left(A \widetilde{\otimes}_{B} A\right) \subseteq A$. And thus the dual of the diagonal lies in $\mathbb{F}$.

\section{$6.3 C^{*}$-Algebras}

In this section we develop functional topology on the opposite Woronowicz category Wor $\left(C^{*} \text {-Alg }\right)^{\text {op }}$ from Section 3, and we discuss the relation with the situation in LcHd. For a morphism $f: A \longrightarrow B$ in Wor $\left(C^{*} \text {-Alg }\right)^{\text {op }}$, we write $F: B \longrightarrow \mathcal{M}(A)$ for the associated Woronowicz-morphism. We make the following definitions for $f: A \longrightarrow B$ :

- $f$ is in $\mathbb{F}$ if $F(B) \subseteq A$;

- $f$ is in $\mathbb{M}=\mathbb{F}_{0}$ if $F(B)=A$;

- $f$ is in $\mathbb{E}$ if $F$ is an isometry.

The following is proven along the lines of Proposition 6.3 (see also [19]):

Proposition $6.4(\mathbb{E} \cap \mathbb{F}, \mathbb{M})$ is a separating $(\mathbb{E}, \mathbb{M})$-proper structure on Wor $\left(C^{*} \text {-Alg }\right)^{\text {op }}$ with $\mathbb{F}$ as proper maps. With respect to the proper class $\mathbb{F}$ :

(1) An object $A$ is compact if and only if it is a unital $C^{*}$-algebra.

(2) An object $A$ is Hausdorff if and only if it is a commutative $C^{*}$-algebra.

(3) All morphisms with commutative domain are separated.

To end this section, we investigate the natural fully faithful functor

$$
\Phi: \mathbf{L c H d} \longrightarrow \operatorname{Wor}\left(C^{*} \text {-Alg }\right)^{\text {op }}: X \longmapsto \mathcal{C}_{0}(X)
$$

which sends a continuous map $f: X \longrightarrow Y$ to the Woronowicz-morphism

$$
\Phi(f)=F: \mathcal{C}_{0}(Y) \longrightarrow \mathcal{C}_{b}(X): g \longrightarrow g \circ f \text {. }
$$


The image of this functor is equivalent to the category $\operatorname{Com}\left(\operatorname{Wor}\left(C^{*}-\mathbf{A l g}\right)^{\mathrm{op}}\right)$ of $R$ commutative objects. Recall from Section 6.1 that LcHd is endowed with the factorization system of dense maps and closed embeddings, and taking the closed immersions equal to the closed embeddings yields a closed structure describing the usual closed maps.

Theorem 6.5 [9] Let $f: X \longrightarrow Y$ be a continuous map between locally compact Hausdorff spaces with associated Woronowicz-morphism $F=\Phi(f)$.

(1) $f$ is proper if and only if $F \in \mathbb{F}$, that is $F\left(\mathcal{C}_{0}(Y)\right) \subseteq \mathcal{C}_{0}(X)$.

(2) $f$ is a closed embedding if and only if $F \in \mathbb{M}$, that is $F\left(\mathcal{C}_{0}(Y)\right)=\mathcal{C}_{0}(X)$.

(3) $f$ is dense if and only if $F \in \mathbb{E}$.

Proof (1) Assume that $f$ is proper and let $g \in \mathcal{C}_{0}(Y)$. For each $\varepsilon>0$, there exists a compact set $K \subseteq Y$ such that $\left\|\left.g\right|_{Y \backslash K}\right\|_{\infty}<\varepsilon$. Since $f$ is proper, we have that $K^{\prime}=f^{-1}(K)$ is a compact set such that $\left\|\left.(g \circ f)\right|_{X \backslash K^{\prime}}\right\|_{\infty}<\varepsilon$. Thus $g \circ f$ vanishes at infinity.

For the converse implication, assume for each $g \in \mathcal{C}_{0}(Y)$ that $g \circ f \in \mathcal{C}_{0}(X)$. Let $K$ be a compact subset of $Y$. By local compactness of $Y$, we know that there exists some compact set $K^{\prime}$ such that $K$ lies in the interior of $K^{\prime}$. Furthermore, $K$ is the intersection of such sets. Now let $g$ be a continuous function such that $g$ is supported in $K^{\prime}$ and such that $g_{K^{\prime}}(K)=1$. Since $g$ is compactly supported and thus lies in $\mathcal{C}_{0}(Y)$, we see that $g \circ f$ lies in $\mathcal{C}_{0}(X)$. Thus there exists a compact set $S \subseteq X$ such that $|g \circ f| \geq 1 / 2$ only on $S$. If $x \in f^{-1}(K)$, then $g_{K^{\prime}}(f(x)) \in K$ and thus $g_{K^{\prime}}(f(x))=1$. Thus $x \in S$. Hence we see that $f^{-1}(K) \subseteq S$. Thus $f^{-1}(K)$ is a closed subset of a compact set and is thus closed.

(2) Assume that $f$ is a closed embedding, then $f$ is certainly proper and thus it follows that $F\left(\mathcal{C}_{0}(Y)\right) \subseteq \mathcal{C}_{0}(X)$. To prove the other inclusion, assume that $g \in \mathcal{C}_{0}(X)$. Then by the Tietze extension theorem (applied on the Alexandroff compactification of $X$ ), we see that $g$ extends to a function $g^{\prime} \in \mathcal{C}_{0}(Y)$. For this function, we clearly have $F\left(g^{\prime}\right)=g$.

For the converse implication, we already know by the previous point that $f$ is proper. It suffices to show that $f$ is injective. So let $x \neq y$ in $X$ such that $f(x)=f(y)$ in $Y$. We can exhibit a function $g \in \mathcal{C}_{0}(X)$ such that $g(x) \neq g(y)$. There exists a function $g^{\prime} \in \mathcal{C}_{0}(Y)$ such that $g^{\prime} \circ f=g$. But then $g(x)=g^{\prime}(f(x))=g^{\prime}(f(y))=g(y)$, a contradiction.

(3) Assume that $f$ is dense. It suffices to prove that $F$ is injective. Thus take $g, g^{\prime} \in \mathcal{C}_{0}(Y)$ such that $g \circ f=g^{\prime} \circ f$. By density of $f$, it follows that $g=g^{\prime}$.

Conversely, assume that $F$ is an isometry. Assume that $X$ is not dense. By local compactness, there then exists a compact subset $K$ of $Y$ with nonempty interior. Let $g$ be a nonzero function supported in $K$. Then $F(g)=0$; while by injectivity of $F$ it follows that $g=0$, a contradiction.

Remark 6.6 From (2) and (3) of the above theorem, it formally follows that $F \in \mathbb{F}$ implies that $f$ is closed (see for instance [16, Proposition 3.48]). In (1), a much more precise result is obtained, namely that $\mathbb{F}$ captures precisely the usual proper maps between locally compact Hausdorff spaces when restricted to the commutative objects.

One could remark that our system of functional topology for $C^{*}$-algebras has $\mathbb{M}$ the closed embeddings and not general embeddings. We now describe more general classes 
which would also encompass usual embeddings. Sadly, we do not yet know whether these classes give rise to a factorization system.

For a morphism $f: A \rightarrow B$ in the category $\operatorname{Wor}\left(C^{*} \text {-Alg }\right)^{o p}$, we write $F: B \rightarrow \mathcal{M}(A)$ for the associated Woronowicz-morphism. We make the following definitions for $f: A \rightarrow$ $B$ :

- $f$ is in $\mathbb{E}^{\prime}$ if $f \in \mathbb{E}$ and if for each pure state $\tau: B \rightarrow \mathbb{C}$ there exists a pure state $\tau^{\prime}: \mathcal{M}(A) \rightarrow \mathbb{C}$ such that $\tau^{\prime} \circ F=\tau$.

- We define the following equivalence relation: we say for $x, y \in B$ that $x \sim_{f} y$ if for each pure state $\tau: B \rightarrow \mathbb{C}$ for which there does not exist a pure state $\tau^{\prime}: \mathcal{M}(A) \rightarrow \mathbb{C}$ with $\tau^{\prime} \circ F=\tau$, we have that $\tau(x)=\tau(y)$.

- We define $f \in \mathbb{O}$ if for each $a \in A$, there exists some $b \in B$ such that $F(b)=a$ and such that $b \sim_{f} 0$.

- Finally, we define $f \in \mathbb{M}^{\prime}$ if its $(\mathbb{E}, \mathbb{M})$ factorization $f=m e$ satisfies $e \in \mathbb{O}$.

This does yield the appropriate notions in the commutative case. So let $f: X \rightarrow Y$ be a continuous map between locally compact Hausdorff spaces, then we get the following Woronowicz-morphism $F: \mathcal{C}_{0}(Y) \rightarrow \mathcal{C}_{b}(X): g \rightarrow g \circ f$. In this case, we get

Theorem 6.7 Under these conditions, we have:

(1) $f$ is surjective if and only if $f$ is in $\mathbb{E}^{\prime}$;

(2) $f$ is a open embedding if and only if $f$ is in $\mathbb{O}$;

(3) $f$ is an embedding if and only if $f$ is in $\mathbb{M}^{\prime}$.

Proof (1) This follows since by Gelfand duality we have that any pure state $\tau: \mathcal{C}_{0}(Y) \rightarrow \mathbb{C}$ has the form $\mathrm{ev}_{y}$, the evaluation in $y$.

(2) By Gelfand duality the pure state $\tau^{\prime}: \mathcal{C}_{0}(X) \rightarrow \mathbb{C}$ corresponds to evaluations ev $v_{x}$ with $x \in X$. Thus $\tau^{\prime} \circ F$ corresponds to the evaluation ev $f(x)$. Thus the pure states $\tau: \mathcal{C}_{0}(Y) \rightarrow \mathbb{C}$ which are not of the form $\tau^{\prime} \circ F$ correspond to evaluations ev $y$ with $y \notin f(X)$. Hence, we see that for $g, g^{\prime} \in \mathcal{C}_{0}(Y)$, we have that $g \sim_{f} g^{\prime}$ if and only if $g(y)=g^{\prime}(y)$ for each $y \notin f(X)$. Thus $g \sim_{f} 0$ if and only if $g$ vanishes outside $f(X)$.

Now let $f$ be an open embedding and let $g \in \mathcal{C}_{0}(X)$. We can extend $g$ by 0 on entire $Y$. Assume that this is not continuous, then there exists some $y \in \partial X$ and some open subset $U$ around $y$ such that $|g| \geq 1 / 2$ on $U \cap X$. But since $g$ vanishes at infinity in $X$, we see that $U \cap X$ must be contained in a compact subset $K$ of $X$. This is also a compact subset of $Y$, and thus $y \in K$. This means that some boundary point of $U$ lies in $K \subseteq X$, this is a contradiction since $X$ was assumed open.

Conversely, let $y$ be a boundary point of $f(X)$ and assume that $y \in f(X)$. Then there exists some $g \in \mathcal{C}_{0}(X)$ such that $g(y)=1$. By hypothesis, for $g$ there exists some $g^{\prime} \in \mathcal{C}_{0}(Y)$ such that $g^{\prime}=g \circ f$ and such that $g^{\prime}$ vanishes outside $f(X)$. This is in contradiction with demanding that $g(y)=1$ for the boundary point $y$. We finally prove that $f$ is injective. To show this, assume that $x \neq y$ in $X$ such that $f(x)=f(y)$. Take $g \in \mathcal{C}_{0}(X)$ such that $g(x) \neq g(y)$. There exists a $g^{\prime} \in \mathcal{C}_{0}(Y)$ such that $g=g^{\prime} f$ and then $g(x)=g^{\prime}(f(x))=g^{\prime}(f(y))=g(y)$, a contradiction. Thus $f$ must have been injective. 
(3) This follows since any locally compact subspace of a locally compact space is an open subspace of a closed subspace.

Acknowledgments The authors wish to thank Venkatesh Chandrasekaran for making suggestions on the English language used in this article.

Open Access This article is distributed under the terms of the Creative Commons Attribution 4.0 International License (http://creativecommons.org/licenses/by/4.0/), which permits unrestricted use, distribution, and reproduction in any medium, provided you give appropriate credit to the original author(s) and the source, provide a link to the Creative Commons license, and indicate if changes were made.

\section{References}

1. Adámek, J., Herrlich, H., Strecker, G.E.: Abstract and concrete categories, Pure and Applied Mathematics (New York). John Wiley \& Sons Inc., New York (1990). The joy of cats, A Wiley-Interscience Publication. MR MR1051419 (91h:18001)

2. Borceux, F.: Handbook of categorical algebra. 2. Categories and structures. Encyclopedia of Mathematics and its Applications, 51. Cambridge University Press, Cambridge (1994). ISBN: 0-521-44179-X. MR MR1313497 (96g:18001b)

3. Bourbaki, N.: Éléments de mathématique. 22. Première partie: Les structures fondamentales de l'analyse. Livre 1: Théorie des ensembles. Chapitre 4: Structures, Actualités Sci. Ind. no. 1258, Hermann, Paris, 1957. MR MR0097335 (20 \#3804)

4. Clementino, M.M., Giuli, E., Tholen, W.: Topology in a category: compactness, Portugal. Math. 53(4), 397-433 (1996). MR 1432147 (97k:54008)

5. Clementino, M.M., Giuli, E., Tholen, W.: A functional approach to general topology. Categorical foundations, Encyclopedia Math. Appl., vol. 97, pp. 103-163. Cambridge University Press, Cambridge (2004). MR 2056582

6. Colebunders, E., Lowen, R., Wuyts, P.: A Kuratowski-Mrówka theorem in approach theory. Topology Appl. 153(5-6), 756-766 (2005). MR 2201487 (2006j:54001)

7. De Commer, K., Van Daele, A.: Multiplier Hopf algebras imbedded in locally compact quantum groups. Rocky Mountain J. Math. 40(4), 11491182 (2010). MR MR2718809 (2011j: 16065)

8. Dikranjan D., Tholen, W.: Categorical structure of closure operators. Mathematics and its Applications, vol. 346. Kluwer Academic Publishers Group, Dordrecht (1995). With applications to topology, algebra and discrete mathematics. MR 1368854 (97i:18004)

9. Gracia-Bondía, J., Várilly, J., Figueroa, H.: Elements of noncommutative geometry. Birkhäuser Advanced Texts: Basler Lehrbücher. Birkhäuser Boston, Inc., Boston, MA (2001). xviii+685 pp. ISBN: 0-8176-4124-6 MR MR1789831 (2001h:58038)

10. Herrlich, H.: A generalization of perfect maps. General topology and its relations to modern analysis and algebra, III (Proc. Third Prague Topological Sympos., 1971), Academia, Prague, 1972, pp. 187-191. MR 0362192 (50 \#14634)

11. Herrlich, H.: Perfect subcategories and factorizations. Topics in topology (Proc. Colloq., Keszthely, 1972), North-Holland, Amsterdam, 1974, pp. 387-403. Colloq. Math. Soc. János Bolyai, Vol. 8. MR 0362193 (50\#14635)

12. Herrlich, H., Salicrup, G., Strecker, G.E.: Factorizations, denseness, separation, and relatively compact objects. In: Proceedings of the 8th international conference on categorical topology (L'Aquila 1986). MR 911689 (89e:18001), vol. 27, pp. 157-169 (1987)

13. Hofmann, D., Tholen, W.: Lax algebra meets topology. Top. Appl. 159(9), 2434-2452 (2012). MR MR2921832

14. Janelidze, Z.: Cover relations on categories. Appl. Categ. Structures 17(4), 351-371 (2009). MR MR2520087 (2010d:18002)

15. Lance, E.C.: Hilbert $C^{*}$-Modules: A toolkit for operator algebraists. London Mathematical Society Lecture Note Series, 210. Cambridge University Press, Cambridge (1995). x+130 pp. ISBN: 0-521-47910-X, MR MR1325694 (96k:46100) 
16. Lowen, W., Mestdagh, J.: Functional topology for geometric settings. J. Pure Appl. Algebra 217(11), 2180-2197 (2013). MR MR3057084

17. Lowen, W., Mestdagh, J.: Woronowicz categories of semigroup objects. In preparation.

18. Manes, E.G.: Compact Hausdorff objects. General Top. Appl. 4, 341-360 (1974). MR 0367901 (51 \#4143)

19. Mestdagh, J.: A study of special morphisms in geometry and analysis. PhD Thesis Universiteit Antwerpen (2015)

20. Penon, J.: Constructions relatives aux objets compacts d'une catégorie. C. R. Acad. Sci. Paris Sér. A-B 275 (1972), A757-A760. MR 0308232 (46 \#7346)

21. Penon, J.: Objets séparés ou compacts dans une catégorie. C. R. Acad. Sci. Paris Sér. A-B 274 (1972), A384-A387. MR 0318256 (47 \#6803)

22. Tholen, W.: A categorical guide to separation, compactness and perfectness. Homology Homotopy Appl. 1, 147-161 (1999). (electronic). MR 1796416 (2002e:18003)

23. Wegge-Olsen, N.E.: $K$-Theory and $C^{*}$-Algebras: A Friendly Approach, Oxford Science Publications. The Clarendon Press, Oxford University Press, New York (1993). xii+370 pp. ISBN: 0-19-859694-4, MR MR1222415 (95c:46116)

24. Woronowicz, S.L.: Compact quantum groups, Symétries quantiques (Les Houches, 1995), 845884, North-Holland, Amsterdam, 1998. MR MR1616348 (99m:46164) 